\title{
Biomimetic Total synthesis of paeoveitol
}

Yuhan Zhang, Yonghong Guo, Zhongle Li, and Zhixiang Xie*

State Key Laboratory of Applied Organic Chemistry \& College of Chemistry and Chemical Engineering, Lanzhou University, Lanzhou 730000, P. R. China

*E-mail: xiezx@lzu.edu.cn.

\section{Table of Contents}

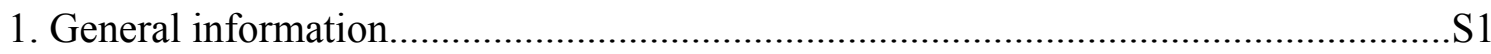

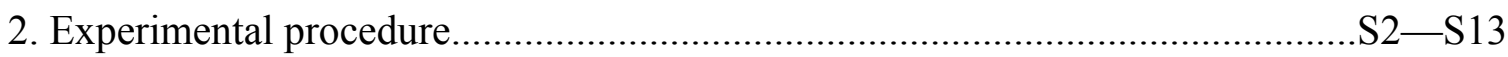

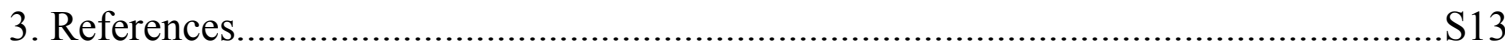

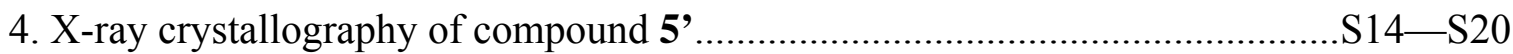

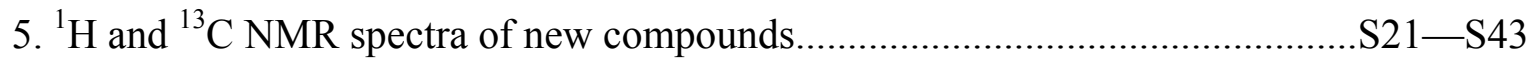

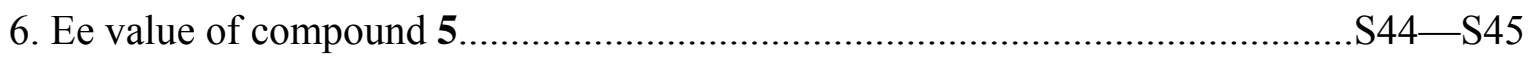

1. General information: Unless otherwise stated, all reactions were performed in oven-dried or flame-dried glassware under an atmosphere of dry nitrogen. Solvents were purified and dried by standard methods prior to use. All commercially available reagents were used without further purification unless otherwise noted. Column chromatography was performed on silica gel (200-300 mesh). NMR spectra were recorded on Bruker 400 $\mathrm{MHz}$ and Oxford $600 \mathrm{MHz}$ spectrometers in the $\mathrm{CDCl}_{3}$ or Pyridine $\mathrm{d}_{5}$ or Methanol $\mathrm{d}_{4}$. Chemical shifts are reported as $\delta$ values relative to internal chloroform $\left(\delta 7.27\right.$ for ${ }^{1} \mathrm{H}$ NMR and 77.00 for ${ }^{13} \mathrm{C}$ NMR $)$ and Methanol $\mathrm{d}_{4}\left(\delta 3.31,4.78\right.$ for ${ }^{1} \mathrm{H}$ NMR and 49.00 for ${ }^{13} \mathrm{C}$ NMR$)$ and Pyridine $\mathrm{d}_{5}\left(\delta 7.22,7.58,8.74\right.$ for ${ }^{1} \mathrm{H}$ NMR and $123.87,135.91,150.35$ for ${ }^{13} \mathrm{C}$ NMR). High resolution mass spectra (HRMS) were obtained on a $4 \mathrm{G}$ mass spectrometer by using electrospray ionization (ESI) analyzed by quadrupole time-of-flight (Q-TOF). Optical rotations were measured on a Rudolph Autoplo IV polarimeter. 


\section{Experimental Procedures}

\section{2,5-Dihydroxy-4-methylacetophenone (2).}<smiles>Cc1cc(O)ccc1O</smiles>

1<smiles>CC(=O)Oc1ccc(OC(C)=O)c(C)c1</smiles>

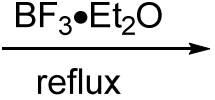

reflux<smiles>CC(=O)c1cc(O)c(C)cc1O</smiles>

2

To a $250 \mathrm{~mL}$ round bottom flask equipped with a stir bar were added 2-methyl benzene-1, 4-diol (1) (3.3 g, $27 \mathrm{mmol}, 1$ equiv) and $\mathrm{CH}_{2} \mathrm{Cl}_{2}(100 \mathrm{~mL})$. This solution was stirred at room temperature, then pyridine $(8.9 \mathrm{~mL}, 0.11 \mathrm{~mol}, 4.0$ equiv) was added dropwise via syringe. After addition of the pyridine, acetyl chloride $(7.5 \mathrm{~mL}, 0.10 \mathrm{~mol}, 3.8$ equiv) was added dropwise via syringe, resulted in a pale yellow mixture. TLC analysis of the reaction mixture showed that the starting material had been consumed, the reaction was quenched with saturated $\mathrm{NH}_{4} \mathrm{Cl}$ (aq.). The resulting mixture was extracted with $\mathrm{CH}_{2} \mathrm{Cl}_{2}$. The organic layer was washed with $\mathrm{CuSO}_{4}$, water followed by brine, and dried over $\mathrm{Na}_{2} \mathrm{SO}_{4}$. It was filtered and concentrated in vacuo. The residue was purified by column chromatography (8:1 petroleum ether/ethyl acetate), provided 2-methyl-1,4-phenylene diacetate as a pale yellow liquid: ( 7.18 g, >99\% ). ${ }^{1} \mathrm{H}$ NMR $\left(400 \mathrm{MHz}, \mathrm{CDCl}_{3}\right) \delta 7.01(\mathrm{~d}, J=8.6 \mathrm{~Hz}, 1 \mathrm{H}), 6.97(\mathrm{~d}, J=2.6 \mathrm{~Hz}, 1 \mathrm{H})$, $6.92(\mathrm{dd}, J=8.6,2.5 \mathrm{~Hz}, 1 \mathrm{H}), 2.31(\mathrm{~s}, 3 \mathrm{H}), 2.28(\mathrm{~s}, 3 \mathrm{H}), 2.17(\mathrm{~s}, 3 \mathrm{H}) .{ }^{13} \mathrm{C} \mathrm{NMR}(100 \mathrm{MHz}$, $\left.\mathrm{CDCl}_{3}\right) \delta 169.4,169.1,148.0,146.7,131.4,123.8,122.6,119.8,21.0,20.7,16.2$.

$24 \mathrm{mmol}$ of 2,5-diacetoxytoluene were added to a solution of $21 \mathrm{~mL}$ (excess 1:5) of boron trifluoride etherate $\left(\mathrm{BF}_{3} \cdot \mathrm{Et}_{2} \mathrm{O}\right)$ and stirred during $4 \mathrm{~h}$ at $130{ }^{\circ} \mathrm{C}$. Then, the solution was cooled to $0{ }^{\circ} \mathrm{C}$ by addition of ice and the mixture extracted with ethyl acetate $(4 \times 30 \mathrm{~mL})$. The combined organic layers was dried over anhydrous $\mathrm{Na}_{2} \mathrm{SO}_{4}$, evaporated and filtered over silica gel to obtain 2 in quantitative yield with no need of further purification. ${ }^{1} \mathrm{H}$ NMR $\left(\mathrm{CD}_{3} \mathrm{OD}, 400 \mathrm{MHz}\right) \delta 7.13(\mathrm{~s}, 1 \mathrm{H}), 6.67(\mathrm{~s}, 1 \mathrm{H}), 2.53(\mathrm{~s}, 3 \mathrm{H}), 2.20(\mathrm{~s}, 3 \mathrm{H}) .{ }^{13} \mathrm{C} \mathrm{NMR}$ $\left(\mathrm{CD}_{3} \mathrm{OD}, 100 \mathrm{MHz}\right) \delta 206.1,157.6,149.8,138.6,121.1,119.6,116.2,27.5,17.8$. IR (neat, $\left.\mathrm{cm}^{-1}\right): v 3320,1706,1200,660$. 


\section{4-hydroxy-5-(1-hydroxyethyl)-2-methylphenyl acetate (3)}

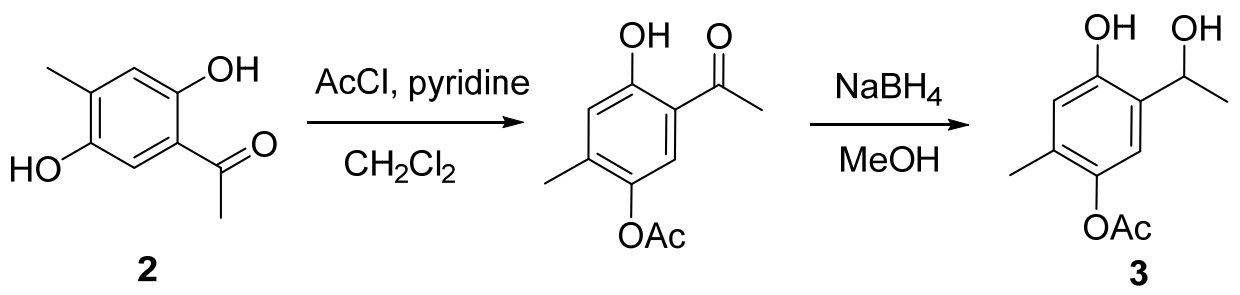

To a $100 \mathrm{~mL}$ round bottom flask equipped with a stir bar were added 2,5-Dihydroxy-4-methylacetophenone (2) (792.0 mg, $4.77 \mathrm{mmol}, 1.0$ equiv) and $\mathrm{CH}_{2} \mathrm{Cl}_{2}$ (47 $\mathrm{mL})$. This solution was stirred at $0{ }^{\circ} \mathrm{C}$, then pyridine $(0.65 \mathrm{~mL}, 8.11 \mathrm{mmol}, 1.7$ equiv) was added dropwise via syringe. After addition of the pyridine, acetyl chloride $(0.47 \mathrm{~mL}, 6.20$ mmol, 1.5 equiv) was added dropwise via syringe, resulted in a pale yellow mixture. After the starting material had been consumed, the reaction was quenched with saturated $\mathrm{NH}_{4} \mathrm{Cl}$ (aq.). The resulting mixture was extracted with $\mathrm{CH}_{2} \mathrm{Cl}_{2}$. The organic layer was washed with $\mathrm{CuSO}_{4}$, water followed by brine, and dried over $\mathrm{Na}_{2} \mathrm{SO}_{4}$. It was filtered and concentrated in vacuo. The residue was purified by column chromatography (8:1 petroleum ether/ethyl acetate), provided 5-acetyl-4-hydroxy-2-methylphenyl acetate as a pale yellow solid ( $893.8 \mathrm{mg}, 90 \%$ ) . ${ }^{1} \mathrm{H}$ NMR $\left(\mathrm{CDCl}_{3}, 400 \mathrm{MHz}\right) \delta 12.10(\mathrm{~s}, 1 \mathrm{H}), 7.35(\mathrm{~s}, 1 \mathrm{H}), 6.84(\mathrm{~s}, 1 \mathrm{H}), 2.56(\mathrm{~s}, 3 \mathrm{H}), 2.32(\mathrm{~s}$, 3H), $2.18(\mathrm{~s}, 3 \mathrm{H}) .{ }^{13} \mathrm{C} \mathrm{NMR}\left(\mathrm{CDCl}_{3}, 100 \mathrm{MHz}\right) \delta 203.3,169.5,160.0,141.1,140.5,122.9$, 120.1, 117.7, 26.4, 20.6, 16.8. IR (neat, $\mathrm{cm}^{-1}$ ): $v$ 3373, 1754, 1628, 1498, 1368, 1225, 1186, 925. HRMS (ESIMS) calcd for $\mathrm{C}_{11} \mathrm{H}_{12} \mathrm{O}_{4} \mathrm{H}^{+}[\mathrm{M}+\mathrm{H}]^{+}$209.0814, found 209.0804.

$\mathrm{NaBH}_{4} \quad(84.0 \mathrm{mg}, 2.22 \mathrm{mmol}, \quad 1.5$ equiv $)$ was added to a solution of 5-acetyl-4-hydroxy-2-methylphenyl acetate $(308.5 \mathrm{mg}, 1.48 \mathrm{mmol}, 1.0$ equiv) in anhydrous methanol $(15 \mathrm{~mL})$ over a period of $5 \mathrm{~min}$ at $0{ }^{\circ} \mathrm{C}$. The reaction mixture was slowly warmed to room temperature and stirred for an additional 0.5 hour. Cold water $(10 \mathrm{~mL})$ was slowly added at $0{ }^{\circ} \mathrm{C}$ and the reaction mixture stirred for $15 \mathrm{~min}$. The aqueous phase was extracted with ethyl acetate $(3 \times 20 \mathrm{~mL})$. The combined organic phases were washed with brine $(50$ $\mathrm{mL}$ ), dried over $\mathrm{Na}_{2} \mathrm{SO}_{4}$, and concentrated in vacuo. The residue was purified by flash column chromatography (16:1 petroleum ether/ethyl acetate) gave $3(258.0 \mathrm{mg}, 83 \%)$ as a pale yellow solid, (m. p. $\left.106 \sim 108^{\circ} \mathrm{C}\right) .{ }^{1} \mathrm{H}$ NMR (400 MHz, MeOD) $\delta 6.91$ (s, 1H), 6.62 (s, 1H), 5.06 (q, $J=6.4 \mathrm{~Hz}, 1 \mathrm{H}), 2.26(\mathrm{~s}, 3 \mathrm{H}), 2.05(\mathrm{~s}, 3 \mathrm{H}), 1.40(\mathrm{~d}, J=6.4 \mathrm{~Hz}, 3 \mathrm{H}) .{ }^{13} \mathrm{C} \mathrm{NMR}(100 \mathrm{MHz}$, MeOD) $\delta 171.7,152.9,143.4,132.0,130.3,120.0,118.0,66.3,24.0,20.6,16.0$. IR (neat, 
$\left.\mathrm{cm}^{-1}\right): v 3393,2975,1755,1735,1370,1219,1179$, 920. HRMS (ESIMS) calcd for $\mathrm{C}_{11} \mathrm{H}_{14} \mathrm{O}_{4} \mathrm{Na}^{+}[\mathrm{M}+\mathrm{Na}]^{+}$233.0790, found 233.0780.

\section{(E)-4-(Prop-1-en-1-yl)morpholine (7)}

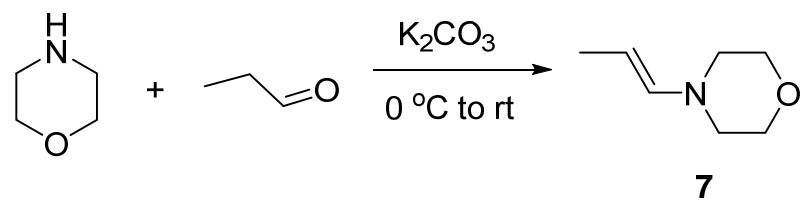

To a stirred mixture of morpholine ( $26 \mathrm{~mL}, 0.294 \mathrm{~mol}, 2.4$ equiv) and anhydrous $\mathrm{K}_{2} \mathrm{CO}_{3}$ (2.0 g, $14.7 \mathrm{mmol}, 117.6$ equiv) was added dropwise propanal ( $9 \mathrm{~mL}, 0.125 \mathrm{~mol}, 1.0$ equiv) at $0{ }^{\circ} \mathrm{C}$. The suspension was stirred at $0{ }^{\circ} \mathrm{C}$ for $2 \mathrm{~h}$ and then warmed to room temperature in about $1 \mathrm{~h}$. The reaction mixture was filtered and washed with dimethyl ether $(2 \times 20 \mathrm{~mL})$. The filtration was concentrated to give a dark color liquid, which was purified by vacuum distillation to give the titled compound 7 as a colorless liquid $(7.5 \mathrm{~g}, 48 \%)$. b. p. $88-90{ }^{\circ} \mathrm{C} / 25$ mm. ${ }^{1} \mathrm{H}$ NMR (400 MHz, $\left.\mathrm{CDCl}_{3}\right) \delta 5.80(\mathrm{~d}, J=15 \mathrm{~Hz}, 1 \mathrm{H}), 4.44(\mathrm{dq}, J=12 \mathrm{~Hz}, 9 \mathrm{~Hz}, 1 \mathrm{H})$, $3.65-3.67(\mathrm{~m}, 4 \mathrm{H}), 2.74-2.76(\mathrm{~m}, 4 \mathrm{H}), 1.61-1.63(\mathrm{~m}, 3 \mathrm{H}) .{ }^{13} \mathrm{C} \mathrm{NMR}\left(100 \mathrm{MHz}, \mathrm{CDCl}_{3}\right) \delta$ $140.1,96.9,66.5,49.5,15.2$.

\section{3,7-dimethylbenzofuran-5-ol (9) and 3,6-dimethylbenzofuran-5-ol (10) ${ }^{2}$}

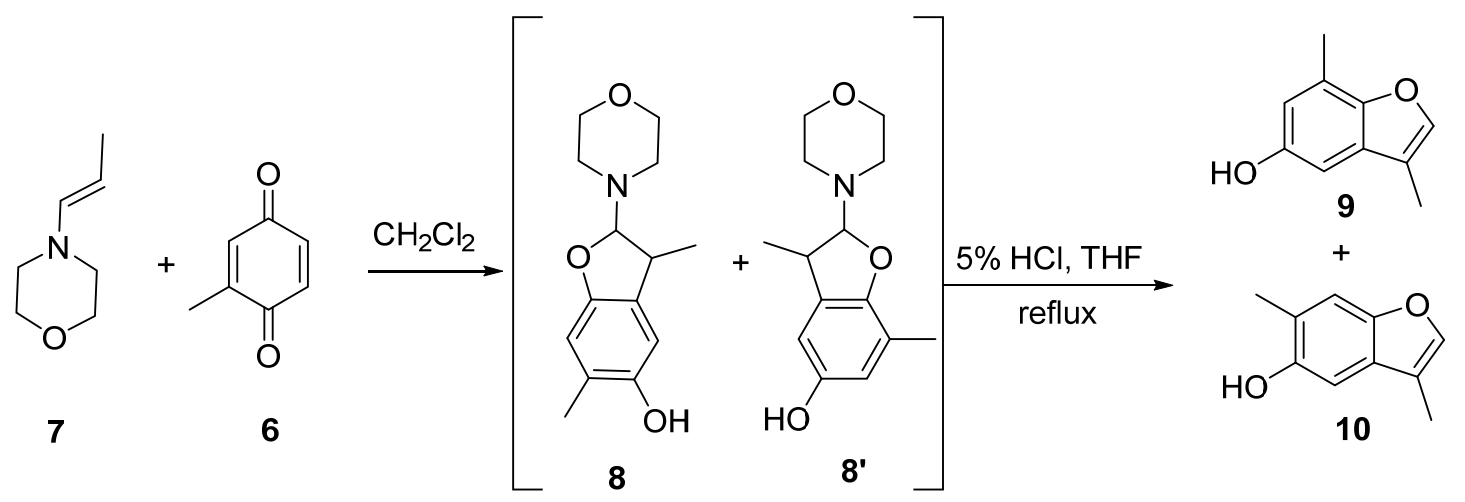

To a solution of methyl-1, 4-benzoquinone (6) (3.6 g, $29.5 \mathrm{mmol}, 1.0$ equiv) in dry $\mathrm{CH}_{2} \mathrm{Cl}_{2}(150 \mathrm{~mL})$ was added $7\left(3.77 \mathrm{~g}, 29.5 \mathrm{mmol}, 1.0\right.$ equiv) at $0{ }^{\circ} \mathrm{C}$. The reaction mixture was then warmed to room temperature and kept for $12 \mathrm{~h}$. Then the solvent was evaporated. The products 8 and 8' were used without further purification. To a solution of $\mathbf{8}$ and 8' (3.5 g, $12.37 \mathrm{mmol}, 0.4$ equiv) in THF $(20 \mathrm{~mL})$ was added diluted hydrochloric acid $(5 \%, 90 \mathrm{~mL})$. The reaction mixture was refluxed until the starting material was consumed, and then cooled 
and extracted by ethyl acetate $(3 \times 30 \mathrm{~mL})$. The organic layers were combined, dried over anhydrous $\mathrm{Na}_{2} \mathrm{SO}_{4}$ and concentrated to give a light pink solid, which was further purified by column chromatography (16:1 petroleum ether/ethyl acetate) to give compound $\mathbf{9}$ as yellow solid (1.01 g, 21\%), (m. p. $\left.96 \sim 98^{\circ} \mathrm{C}\right) .{ }^{1} \mathrm{H}$ NMR (400 MHz, $\left.\mathrm{CDCl}_{3}\right) \delta 7.38(\mathrm{~s}, 1 \mathrm{H}), 6.75(\mathrm{~d}$, $J=2.2 \mathrm{~Hz}, 1 \mathrm{H}), 6.63(\mathrm{~s}, 1 \mathrm{H}), 4.69(\mathrm{~s}, 1 \mathrm{H}), 2.45(\mathrm{~s}, 3 \mathrm{H}), 2.17(\mathrm{~s}, 3 \mathrm{H}) .{ }^{13} \mathrm{C} \mathrm{NMR}(100 \mathrm{MHz}$, $\left.\mathrm{CDCl}_{3}\right) \delta 151.1,149.3,142.1,129.2,122.3,115.6,113.5,101.7,15.0,8.0$. IR (neat, $\mathrm{cm}^{-1}$ ): $v$ 3346, 1608, 1265, 1200, 1096, 836. HRMS (ESIMS) calcd for $\mathrm{C}_{10} \mathrm{H}_{10} \mathrm{O}_{2} \mathrm{H}^{+}[\mathrm{M}+\mathrm{H}]^{+}$ 163.0759, found 163.0751. And compound 10 as white solid (4.8 g, 72\%), (m. p. 119 $\left.120{ }^{\circ} \mathrm{C}\right) .{ }^{1} \mathrm{H}$ NMR $\left(400 \mathrm{MHz}, \mathrm{CDCl}_{3}\right) \delta 7.31(\mathrm{~s}, 1 \mathrm{H}), 7.21(\mathrm{~s}, 1 \mathrm{H}), 6.87(\mathrm{~s}, 1 \mathrm{H}), 4.66(\mathrm{~d}, J=$ $7.0 \mathrm{~Hz}, 1 \mathrm{H}), 2.35$ (s, 3H), 2.17 (s, 3H). $\left.{ }^{13} \mathrm{C} \mathrm{NMR} \mathrm{(101} \mathrm{MHz,} \mathrm{CDCl}_{3}\right) \delta 150.2,149.7,141.5$, 127.6, 121.3, 115.2, 112.6, 103.9, 16.6, 8.0. IR (neat, $\mathrm{cm}^{-1}$ ): v 3257, 1600, 1439, 1165, 1087, 852, 837, 792. HRMS (ESIMS) calcd for $\mathrm{C}_{10} \mathrm{H}_{10} \mathrm{O}_{2} \mathrm{H}^{+}[\mathrm{M}+\mathrm{H}]^{+}$163.0759, found 163.0752.

\section{3,6-dimethylbenzofuran-5-yl acetate (11)}

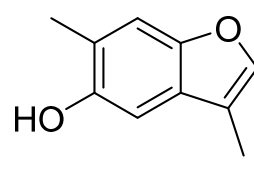

10

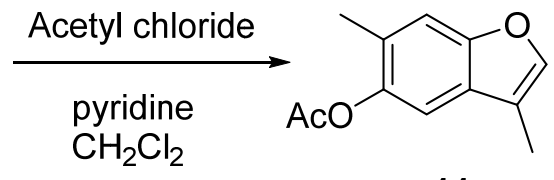

11

To a $25 \mathrm{~mL}$ round bottom flask equipped with a stir bar were added 3,6-dimethylbenzofuran-5-ol (10) (224.9 mg, $1.39 \mathrm{mmol}, 1.0$ equiv) and $\mathrm{CH}_{2} \mathrm{Cl}_{2}$ (15 ml). This solution was stirred at $0{ }^{\circ} \mathrm{C}$, then pyridine $(0.2 \mathrm{~mL}, 2.64 \mathrm{mmol}, 1.9$ equiv) was added dropwise via syringe. After addition of the pyridine, acetyl chloride $(0.16 \mathrm{~mL}, 2.09 \mathrm{mmol}, 1.5$ equiv) was added dropwise via syringe, resulted in a pale yellow mixture. After the starting material had been consumed, the reaction was quenched with saturated $\mathrm{NH}_{4} \mathrm{Cl}$ (aq.). The resulting mixture was extracted with $\mathrm{CH}_{2} \mathrm{Cl}_{2}(3 \times 30 \mathrm{~mL})$. The organic layer was washed with $\mathrm{CuSO}_{4}$, water followed by brine, and dried over $\mathrm{Na}_{2} \mathrm{SO}_{4}$. It was filtered and concentrated in vacuo. The residue was purified by column chromatography (16:1 petroleum ether/ethyl acetate), provided 3,6-dimethylbenzofuran-5-yl acetate (11) as a pale yellow oil (276.9 mg, 98\%). ${ }^{1} \mathrm{H}$ NMR (400 MHz, $\left.\mathrm{CDCl}_{3}\right) \delta 7.37$ (d, $\left.J=1.0 \mathrm{~Hz}, 1 \mathrm{H}\right), 7.31(\mathrm{~s}, 1 \mathrm{H}), 7.16(\mathrm{~s}, 1 \mathrm{H}), 2.36$ (s, 3H), $2.29(\mathrm{~s}, 3 \mathrm{H}), 2.20(\mathrm{~d}, J=1.1 \mathrm{~Hz}, 3 \mathrm{H}) .{ }^{13} \mathrm{C} \mathrm{NMR}\left(100 \mathrm{MHz}, \mathrm{CDCl}_{3}\right) \delta 169.8,153.1$, 145.0, 141.9, 127.7, 126.5, 115.7, 112.9, 112.0, 20.8, 16.8, 7.9. IR (neat, $\mathrm{cm}^{-1}$ ): v 3453, 2926, 
1805, 1760, 1475, 1369, 1210, 913. HRMS (ESIMS) calcd for $\mathrm{C}_{12} \mathrm{H}_{12} \mathrm{O}_{3} \mathrm{H}^{+}[\mathrm{M}+\mathrm{H}]^{+}$ 205.0865, found 205.0855.

\section{Paeoveitol D}<smiles>CC(=O)Oc1cc2c(C)coc2cc1C</smiles>

11

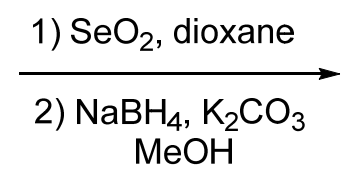

$\mathrm{MeOH}$<smiles>Cc1cc2occ(CO)c2cc1O</smiles>

Paeoveitol D

The solution of 3,6-dimethylbenzofuran-5-yl acetate (11) $(276.1 \mathrm{mg}, 1.35 \mathrm{mmol}, 1.0$ equiv) in dioxane $(17 \mathrm{~mL})$, heated to $100{ }^{\circ} \mathrm{C}$, added $\mathrm{SeO}_{2}(179.8 \mathrm{mg}, 1.62 \mathrm{mmol}, 1.2$ equiv), then the mixture refluxed over $16 \mathrm{~h}$. Cooled to room temperature, the crude product was filtered, the residue washed with THF $(20 \mathrm{~mL} \times 3)$, and the solvent was evaporated. After the mixture resolved in anhydrous methanol $(20 \mathrm{~mL}), \mathrm{NaBH}_{4}(76.0 \mathrm{mg}, 2.0 \mathrm{mmol}, 1.5$ equiv) was added at $0{ }^{\circ} \mathrm{C}$. As long as the starting material was consumed, the anhydrous $\mathrm{K}_{2} \mathrm{CO}_{3}$ (373.2 $\mathrm{mg}, 2.70 \mathrm{mmol}, 2.0$ equiv) was added. The progress of reaction was monitored by TLC. The reaction mixture was quenched with saturated $\mathrm{NH}_{4} \mathrm{Cl}$ (aq.), then extracted with ethyl acetate $(3 \times 30 \mathrm{~mL})$, water followed by brine, and dried over anhydrous $\mathrm{Na}_{2} \mathrm{SO}_{4}$. It was filtered and concentrated in vacuo. The residue was purified by column chromatography $(2: 1$ petroleum ether/ethyl acetate), provided paeoveitol D as a yellow solid (322.3 $\mathrm{mg}, 77 \%, 84 \%$ brsm) and 10 (17.8 mg, $0.11 \mathrm{mmol})$. paeoveitol D. ${ }^{1} \mathrm{H}$ NMR (400 MHz, MeOD) $\delta 7.54(\mathrm{~s}, 1 \mathrm{H})$, $7.16(\mathrm{~s}, 1 \mathrm{H}), 6.99$ (s, 1H), 4.67 (s, 2H), 2.28 (s, 3H). ${ }^{13} \mathrm{C}$ NMR (100 MHz, MeOD) $\delta$ 152.6, 151.6, 143.3, 126.6, 124.0, 121.6, 113.3, 104.8, 55.8, 17.1. IR (neat, $\mathrm{cm}^{-1}$ ): $v$ 3488, 2581, 2077, 1468, 1436, 1137, 989, 856.

(5aR,10aR,11R)-3,11-dimethyl-5a,10a-dihydro-11H-benzofuro[3,2-b]chromen-2-yl acetate (5)

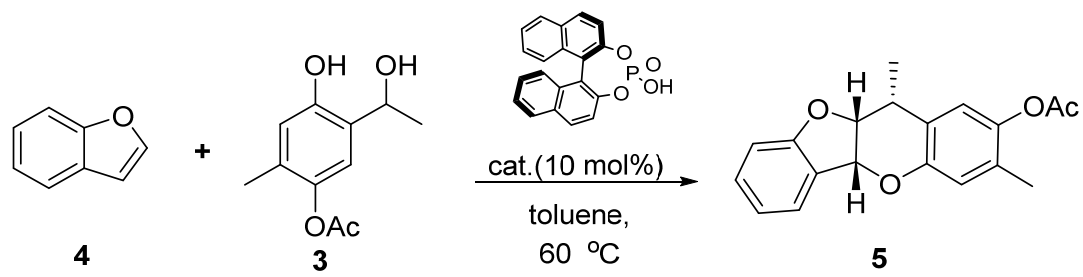

A $5 \mathrm{~mL}$ bottom was charged with Benzofuran (4) $(52 \mu \mathrm{L}, 0.52 \mathrm{mmol}, 4.0$ equiv), 
4-hydroxy-5-(1-hydroxyethyl)-2-methylphenyl acetate (3) (26.4 mg, $0.13 \mathrm{mmol}, 1.0$ equiv), (R)1,1'-Binaphthyl-2,2'-diyl hydrogenphosphate (4.5 mg, $0.01 \mathrm{mmol}, 0.08$ equiv) and toluene $(1 \mathrm{~mL})$. The mixture heated at $60{ }^{\circ} \mathrm{C}$ for $24 \mathrm{~h}$ in a drying oven. The resulting reaction mixture was cooled to ambient temperature then, the solvent evaporated, and the residue was purified by column chromatography (10:1 petroleum ether/ethyl acetate) gave compound 5 (25.8 $\mathrm{mg}$, $64 \%$ ) as a white solid, (m. p. $\left.145 \sim 147^{\circ} \mathrm{C}\right) .[\alpha]^{20.0}{ }_{\mathrm{D}}+9.0\left(c 1.00, \mathrm{CHCl}_{3}\right) .{ }^{1} \mathrm{H}$ NMR $(400 \mathrm{MHz}$, $\left.\mathrm{CDCl}_{3}\right) \delta 7.46(\mathrm{~d}, J=7.4 \mathrm{~Hz}, 1 \mathrm{H}), 7.16(\mathrm{t}, J=8.4 \mathrm{~Hz}, 1 \mathrm{H}), 6.89(\mathrm{t}, J=7.4 \mathrm{~Hz}, 1 \mathrm{H}), 6.83(\mathrm{~s}$, $1 \mathrm{H}), 6.65(\mathrm{~d}, J=8.1 \mathrm{~Hz}, 1 \mathrm{H}), 6.58(\mathrm{~s}, 1 \mathrm{H}), 5.84(\mathrm{~d}, J=7.7 \mathrm{~Hz}, 1 \mathrm{H}), 5.12(\mathrm{dd}, J=7.7,3.2 \mathrm{~Hz}$, $1 \mathrm{H}), 3.17(\mathrm{dd}, J=6.8,2.7 \mathrm{~Hz}, 1 \mathrm{H}), 2.27(\mathrm{~s}, 3 \mathrm{H}), 2.00(\mathrm{~s}, 3 \mathrm{H}), 1.58(\mathrm{~d}, J=7.0 \mathrm{~Hz}, 3 \mathrm{H}) .{ }^{13} \mathrm{C}$ NMR $\left(100 \mathrm{MHz}, \mathrm{CDCl}_{3}\right) \delta 169.5,160.6,151.6,144.3,130.9,128.9,127.0,126.2,125.9$, 120.8, 120.0, 119.3, 110.0, 86.0, 79.5, 31.4, 20.7, 15.8, 13.5. IR (neat, $\mathrm{cm}^{-1}$ ): v 3375, 2962, 2928, 1738, 1499, 1480, 1372, 1240, 1215, 1159, 753. HRMS (ESIMS) calcd for $\mathrm{C}_{19} \mathrm{H}_{18} \mathrm{O}_{4} \mathrm{Na}^{+}[\mathrm{M}+\mathrm{Na}]^{+}$333.1103, found 333.1092.

\section{3,11-dimethyl-5a,10a-dihydro-11H-benzofuro[3,2-b]chromen-2-ol (5a')}

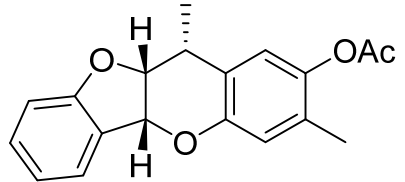

5
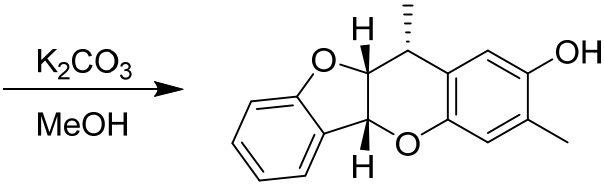

$\mathbf{5}^{\prime}$

To a $5 \mathrm{~mL}$ round bottom flask equipped with a stir bar were added compound 5 (10.6 $\mathrm{mg}$, 0.034 mmol, 1.0 equiv) and $\mathrm{CH}_{3} \mathrm{OH}(1 \mathrm{~mL})$. The solution was stirred at room temperature, the anhydrous $\mathrm{K}_{2} \mathrm{CO}_{3}(4.7 \mathrm{mg}, 0.034 \mathrm{mmol}, 1.0$ equiv) was added. The progress of reaction was monitored by TLC. The reaction was quenched with saturated $\mathrm{NH}_{4} \mathrm{Cl}$ (aq.). The resulting mixture was extracted with ethyl acetate $(3 \times 20 \mathrm{~mL})$. The organic layer was washed with water followed by brine, and dried over anhydrous $\mathrm{Na}_{2} \mathrm{SO}_{4}$. It was filtered and concentrated in vacuo. The residue was purified by column chromatography (4:1 petroleum ether/ethyl acetate) provided 5' (6.9 mg, 77\%) as a yellow solid. (m. p. $\left.179 \sim 181{ }^{\circ} \mathrm{C}\right) .{ }^{1} \mathrm{H}$ NMR $(400$ MHz, MeOD) $\delta 7.42-7.36(\mathrm{~m}, 1 \mathrm{H}), 7.13-7.06(\mathrm{~m}, 1 \mathrm{H}), 6.83(\mathrm{td}, \mathrm{J}=7.4,0.8 \mathrm{~Hz}, 1 \mathrm{H}), 6.60$ (s, 1H), 6.55 (d, J = 8.1 Hz, 1H), $6.34(\mathrm{~s}, 1 \mathrm{H}), 5.79$ (d, J = $7.7 \mathrm{~Hz}, 1 \mathrm{H}), 5.12(\mathrm{dd}, \mathrm{J}=7.7,3.1$ $\mathrm{Hz}, 1 \mathrm{H}), 3.13-3.04(\mathrm{~m}, 1 \mathrm{H}), 2.00(\mathrm{~d}, \mathrm{~J}=7.3 \mathrm{~Hz}, 1 \mathrm{H}), 1.53(\mathrm{~d}, \mathrm{~J}=7.0 \mathrm{~Hz}, 1 \mathrm{H}) .{ }^{13} \mathrm{C} \mathrm{NMR}$ 
$(100 \mathrm{MHz}, \mathrm{MeOD}) \delta 162.1,151.5,147.9,131.7,128.5,127.6,127.3,123.9,121.6,120.7$, 113.3, 110.5, 87.9, 80.8, 32.7, 15.8, 13.8. HRMS (ESIMS) calcd for $\mathrm{C}_{17} \mathrm{H}_{16} \mathrm{O}_{3} \mathrm{~K}^{+}[\mathrm{M}+\mathrm{K}]^{+}$ 307.0734, found 307.0731.

(2R,3S)-5'-hydroxy-3,5,6'-trimethyl-3'-methylene-3H,3'H-2,2'-spirobi[benzofuran]-6-yl acetate (12)
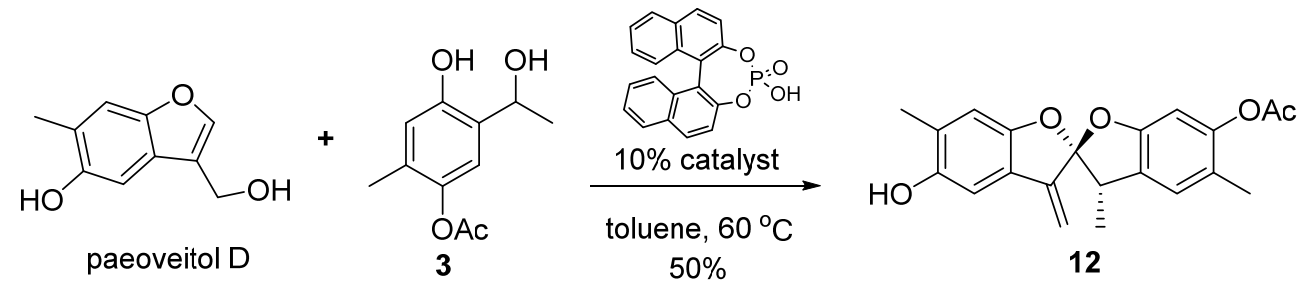

Specific steps for the operation of reference compounds 5. Purified by column chromatography (4:1 petroleum ether/ethyl acetate) gave compound 12 (50\%) as a yellow oil. ${ }^{1} \mathrm{H}$ NMR (400 MHz, $\left.\mathrm{CDCl}_{3}\right) \delta 6.88(\mathrm{~s}, 1 \mathrm{H}), 6.84(\mathrm{~s}, 1 \mathrm{H}), 6.74(\mathrm{~s}, 1 \mathrm{H}), 6.65(\mathrm{~s}, 1 \mathrm{H}), 5.68(\mathrm{~s}$, 1H), $5.36(\mathrm{~s}, 1 \mathrm{H}), 4.51(\mathrm{~s}, 1 \mathrm{H}), 3.62(\mathrm{q}, J=7.2 \mathrm{~Hz}, 1 \mathrm{H}), 2.33(\mathrm{~s}, 3 \mathrm{H}), 2.25(\mathrm{~s}, 3 \mathrm{H}), 2.15$ (s, $3 \mathrm{H}), 1.30(\mathrm{~d}, J=6.8 \mathrm{~Hz}, 3 \mathrm{H}) .{ }^{13} \mathrm{C} \mathrm{NMR}\left(100 \mathrm{MHz}, \mathrm{CDCl}_{3}\right) \delta 169.8,154.7,153.9,148.7$, $144.8,143.8,130.1,128.9,127.9,122.3,120.3,116.9,112.1,111.7,107.0,106.6,46.8,20.8$, 16.6, 16.5, 11.0. IR (neat, $\mathrm{cm}^{-1}$ ): $v$ 3431, 2971, 2932, 2254, 1750, 1463, 1370, 1195, 912, 733, 649. HRMS (ESIMS) calcd for $\mathrm{C}_{21} \mathrm{H}_{20} \mathrm{O}_{5} \mathrm{Na}^{+}[\mathrm{M}+\mathrm{Na}]^{+} 353.1389$, found 353.1384.

\section{4-hydroxy-5-(1-(5-hydroxy-3,6-dimethylbenzofuran-2-yl)ethyl)-2-methylphenyl acetate} (13)<smiles>Cc1cc2occ(C)c2cc1O</smiles>

10<smiles>CC(=O)Oc1cc(C(C)O)c(O)cc1[18O]</smiles>

3

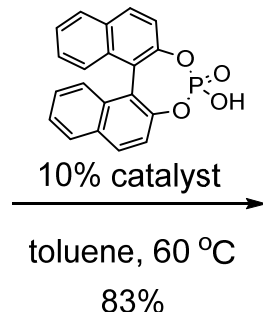

$83 \%$

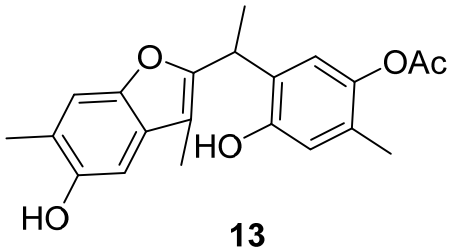

13

Specific steps for the operation of reference compounds 5. Purified by column chromatography (10:1 petroleum ether/ethyl acetate) gave compound $13(83 \%)$ as a yellow solid, (m. p. $\left.159 \sim 163{ }^{\circ} \mathrm{C}\right) .{ }^{1} \mathrm{H}$ NMR $\left(400 \mathrm{MHz}, \mathrm{CDCl}_{3}\right) \delta 7.14$ (s, 1H), 6.90 (s, 1H), 6.73 (s, 
1H), $6.57(\mathrm{~s}, 1 \mathrm{H}), 5.52(\mathrm{~s}, 1 \mathrm{H}), 4.80(\mathrm{~s}, 1 \mathrm{H}), 4.48(\mathrm{q}, J=7.3 \mathrm{~Hz}, 1 \mathrm{H}), 2.30(\mathrm{~s}, 3 \mathrm{H}), 2.28$ (s, 3H), 2.05 (s, 3H), 2.04 (s, 3H), 1.63(d, $J=7.3 \mathrm{~Hz}, 3 \mathrm{H}) .{ }^{13} \mathrm{C} \mathrm{NMR}\left(100 \mathrm{MHz}, \mathrm{CDCl}_{3}\right) \delta 169.9$, $154.7,150.8,149.7,148.7,142.8,129.2,128.9,128.2,121.5,120.5,118.2,112.2,109.5$, $103.8,31.8,20.8,18.4,16.5,15.8,7.9$. IR (neat, $\mathrm{cm}^{-1}$ ): $v 3431,2929,1736,1461,1421,1371$, 1184, 915, 734. HRMS (ESIMS) calcd for $\mathrm{C}_{21} \mathrm{H}_{22} \mathrm{O}_{5} \mathrm{Na}^{+}[\mathrm{M}+\mathrm{Na}]^{+}$377.1365, found 377.1354 .

\section{2-(1-hydroxyethyl)-5-methyl-4-((triethylsilyl)oxy)phenol (14)}

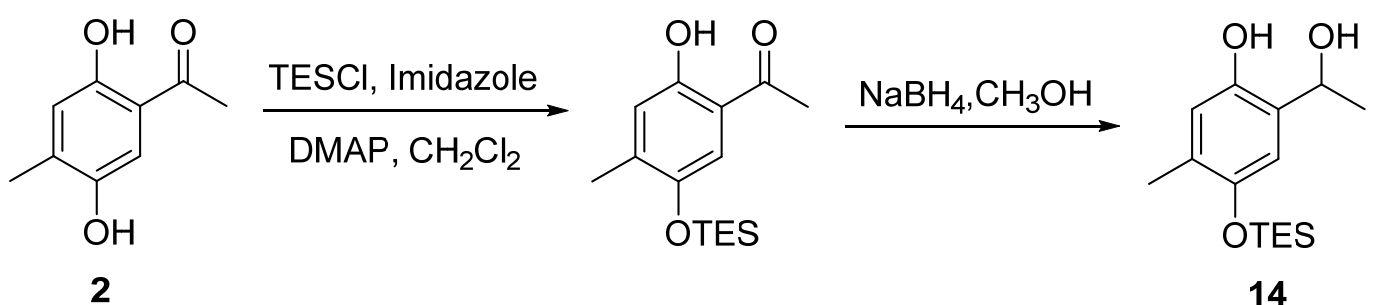

To a $5 \mathrm{~mL}$ round bottom flask equipped with a stir bar were added 5-Dihydroxy-4-methylacetophenone (2) (35.8 mg, $0.22 \mathrm{mmol}, 1.0$ equiv), DMAP (5.4 mg, $0.044 \mathrm{mmol}, 0.2$ equiv) and imidazole $(45.0 \mathrm{mg}, 0.66 \mathrm{mmol}, 3.0$ equiv) and resolved in $\mathrm{CH}_{2} \mathrm{Cl}_{2}(3 \mathrm{~mL})$. The solution was stirred at room temperature, then TESCl $(0.043 \mathrm{~mL}, 0.26$ mmol, 1.2 equiv) was added dropwise via syringe. The progress of reaction was monitored by TLC. After the starting material had been consumed, the reaction was quenched with saturated $\mathrm{NH}_{4} \mathrm{Cl}$ (aq.). The resulting mixture was extracted with $\mathrm{CH}_{2} \mathrm{Cl}_{2}$. The organic layer was washed with water followed by brine, and dried over anhydrous $\mathrm{Na}_{2} \mathrm{SO}_{4}$. It was filtered and concentrated in vacuo. The residue was purified by column chromatography (50:1 petroleum ether/ethyl acetate) provided the product $(55.0 \mathrm{mg}, 89 \%)$ as a yellow oil. ${ }^{1} \mathrm{H}$ NMR $\left(400 \mathrm{MHz}, \mathrm{CDCl}_{3}\right) \delta 11.86(\mathrm{~s}, 1 \mathrm{H}), 7.05(\mathrm{~s}, 1 \mathrm{H}), 6.75(\mathrm{~s}, 1 \mathrm{H}), 2.54(\mathrm{~s}, 3 \mathrm{H}), 2.22(\mathrm{~s}, 3 \mathrm{H}), 1.01$ $(\mathrm{t}, J=7.9 \mathrm{~Hz}, 9 \mathrm{H}), 0.76(\mathrm{q}, 6 \mathrm{H}) .{ }^{13} \mathrm{C} \mathrm{NMR}\left(100 \mathrm{MHz}, \mathrm{CDCl}_{3}\right) \delta 203.2,156.8,146.0,140.3$, 119.8, 118.0, 117.4, 26.4, 17.4, 6.6, 5.2. IR (neat, $\mathrm{cm}^{-1}$ ): v 3255, 2957, 2878, 1646, 1493, 1324, 1218,900 .

$\mathrm{NaBH}_{4} \quad(4.0 \mathrm{mg}, \quad 0.065$ mmol, 1.5 equiv $)$ was added to a solution of 1-(2-hydroxy-4-methyl-5-((triethylsilyl)oxy)phenyl)ethan-1-one (18.2 mg, $0.065 \mathrm{mmol}, 1.0$ equiv) in anhydrous methanol $(2 \mathrm{~mL})$ over a period of $5 \mathrm{~min}$ at room temperature. And the reaction mixture stirred for $15 \mathrm{~min}$. The progress of reaction was monitored by TLC. After the 
starting material had been consumed, the reaction was quenched with water. The aqueous phase was extracted with ethyl acetate $(3 \times 15 \mathrm{~mL})$. The combined organic phases were washed with brine $(30 \mathrm{~mL})$, dried over $\mathrm{Na}_{2} \mathrm{SO}_{4}$, and concentrated in vacuo. The residue was purified by flash column chromatography (4:1 petroleum ether/ethyl acetate) gave compound $14\left(14.0 \mathrm{mg}, 76 \%\right.$ ) as a white solid, (m. p. $\left.63 \sim 65{ }^{\circ} \mathrm{C}\right) .{ }^{1} \mathrm{H}$ NMR $\left(400 \mathrm{MHz}, \mathrm{CDCl}_{3}\right) \delta 7.32$ (s, 1H), $6.64(\mathrm{~s}, 1 \mathrm{H}), 6.39$ (s, 1H), $4.96(\mathrm{dd}, J=6.2,3.0 \mathrm{~Hz}, 1 \mathrm{H}), 2.40(\mathrm{~s}, 1 \mathrm{H}), 2.14(\mathrm{~s}, 1 \mathrm{H}), 1.55$ $(\mathrm{d}, J=6.6 \mathrm{~Hz}, 1 \mathrm{H}), 0.98(\mathrm{t}, J=7.9 \mathrm{~Hz}, 1 \mathrm{H}), 0.72(\mathrm{q}, J=7.9 \mathrm{~Hz}, 1 \mathrm{H}) .{ }^{13} \mathrm{C}$ NMR $(100 \mathrm{MHz}$, $\left.\mathrm{CDCl}_{3}\right) \delta 148.9,146.7,129.0,126.3,119.0,116.3,71.0,23.3,16.3,6.7,5.2$. IR (neat, $\left.\mathrm{cm}^{-1}\right): v$ $3367,2957,2878,1501,1415,1195,903,736$. HRMS (ESIMS) calcd for $\mathrm{C}_{15} \mathrm{H}_{26} \mathrm{O}_{3} \mathrm{SiNa}^{+}[\mathrm{M}$ $+\mathrm{Na}]^{+} 305.1549$, found 305.1543 .

(2R,3'S)-3',5',6-trimethyl-3-methylene-6'-((triethylsilyl)oxy)-3H,3'H-2,2'-spirobi[benzofu ran]-5-ol (15)

\section{3,6,9-trimethyl-8-((triethylsilyl)oxy)-6,12-dihydrobenzo[6,7]oxepino[4,3-b]benzofuran-2- ol (16)}

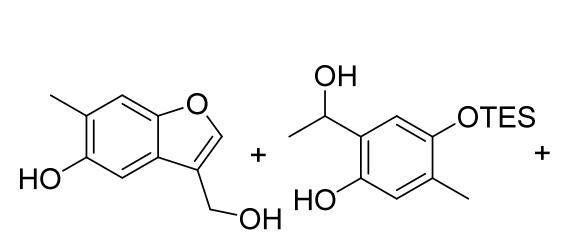

Paeoveitol D

14
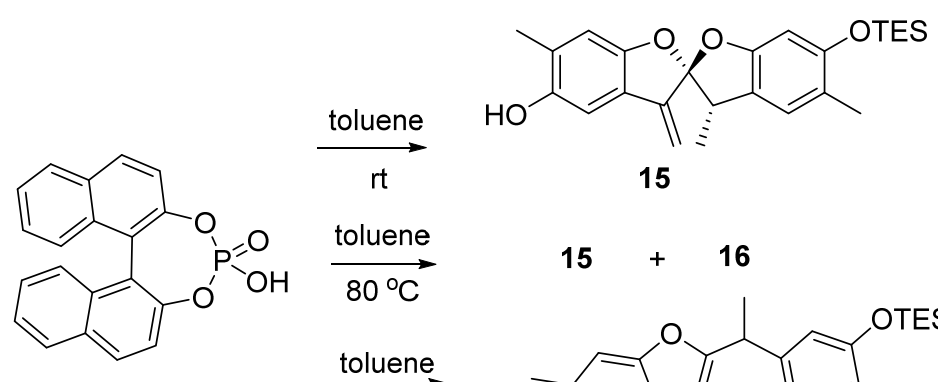

$\underset{80{ }^{\circ} \mathrm{C}}{\stackrel{\text { toluene }}{\longrightarrow}}$

$\underset{120^{\circ} \mathrm{C}}{\stackrel{\text { toluene }}{\longrightarrow}}$

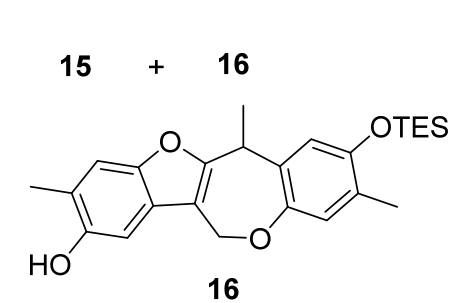

A $5 \mathrm{ml}$ bottle was charged with paeoveitol $\mathrm{D}(8.5 \mathrm{mg}, 0.04 \mathrm{mmol}, 1.0$ equiv), 2-(1-hydroxyethyl)-5-methyl-4-((triethylsilyl)oxy) phenol (14) (11.0 mg, $0.04 \mathrm{mmol}, 1.0$ equiv), 1,1'-Binaphthyl-2,2'-diyl hydrogenphosphate (1.4 mg, $0.004 \mathrm{mmol}, 0.1$ equiv) and toluene $(1 \mathrm{~mL})$. The bottle stirred at room temperature for $24 \mathrm{~h}$ in a drying oven. The resulting reaction mixture was purified by column chromatography ( $8: 1$ petroleum ether/ethyl acetate) give compound $15(8.5 \mathrm{mg}, 50 \%)$ as a brown oil. ${ }^{1} \mathrm{H}$ NMR $\left(400 \mathrm{MHz}, \mathrm{CDCl}_{3}\right) \delta 6.87(\mathrm{~s}, 1 \mathrm{H})$, $6.66(\mathrm{~s}, 1 \mathrm{H}), 6.64(\mathrm{~s}, 1 \mathrm{H}), 6.57(\mathrm{~s}, 1 \mathrm{H}), 5.66(\mathrm{~s}, 1 \mathrm{H}), 5.37(\mathrm{~s}, 1 \mathrm{H}), 4.45(\mathrm{~s}, 1 \mathrm{H}), 3.57$ (q, $J=6.9$ $\mathrm{Hz}, 1 \mathrm{H}), 2.23$ (s, 3H), 2.18 (s, 3H), 1.28 (d, $J=7.1 \mathrm{~Hz}, 3 \mathrm{H}), 1.01$ (t, $J=7.9 \mathrm{~Hz}, 9 \mathrm{H}), 0.76$ (q, 
$J=7.9 \mathrm{~Hz}, 6 \mathrm{H}) .{ }^{13} \mathrm{C} \mathrm{NMR}\left(100 \mathrm{MHz}, \mathrm{CDCl}_{3}\right) \delta 154.1,150.9,148.9,148.5,145.1,128.4$, 128.0, 127.8, 122.5, 120.2, 113.4, 112.1, 111.7, 106.9, 106.2, 47.3, 17.0, 16.6, 11.2, 6.7, 5.3. IR (neat, $\mathrm{cm}^{-1}$ ): $v$ 3377, 2958, 2925, 1499, 1460, 1199, 909, 734. HRMS (ESIMS) calcd for $\mathrm{C}_{25} \mathrm{H}_{32} \mathrm{O}_{4} \mathrm{SiH}^{+}[\mathrm{M}+\mathrm{H}]^{+}$425.2070, found 425.2138.

General procedure followed, but the temperature control at $120{ }^{\circ} \mathrm{C}$. The resulting reaction mixture was purified by column chromatography (8:1 petroleum ether/ethyl acetate) gave compound 16 (8.6 mg, 51\%) as a brown oil. ${ }^{1} \mathrm{H}$ NMR $\left(400 \mathrm{MHz}, \mathrm{CDCl}_{3}\right) \delta 7.17(\mathrm{~s}, 1 \mathrm{H})$, $6.94(\mathrm{~s}, 1 \mathrm{H}), 6.65(\mathrm{~s}, 1 \mathrm{H}), 6.57(\mathrm{~s}, 1 \mathrm{H}), 5.22(\mathrm{~d}, J=14.6 \mathrm{~Hz}, 1 \mathrm{H}), 4.84(\mathrm{~d}, J=14.6 \mathrm{~Hz}, 1 \mathrm{H})$, $4.54(\mathrm{~d}, J=4.4 \mathrm{~Hz}, 1 \mathrm{H}), 3.96(\mathrm{q}, J=7.2 \mathrm{~Hz}, 1 \mathrm{H}), 2.33(\mathrm{~s}, 3 \mathrm{H}), 2.17(\mathrm{~s}, 3 \mathrm{H}), 1.64(\mathrm{~d}, J=7.1$ $\mathrm{Hz}, 3 \mathrm{H}), 0.99(\mathrm{t}, J=7.9 \mathrm{~Hz}, 9 \mathrm{H}), 0.75(\mathrm{q}, J=7.9 \mathrm{~Hz}, 6 \mathrm{H}) \cdot{ }^{13} \mathrm{C} \mathrm{NMR}\left(100 \mathrm{MHz}, \mathrm{CDCl}_{3}\right) \delta$ $155.4,151.4,150.4,149.7,148.7,134.8,128.3,125.9$, 124.9, 120.5 , 118.3, 112.3, 111.9, 103.2, 68.1, 38.9, 22.3, 16.5, 16.2, 6.7, 5.3. IR (neat, $\mathrm{cm}^{-1}$ ): $v$ 3402, 2958, 2925, 1499, 1460, 1197, 909, 734. HRMS (ESIMS) calcd for $\mathrm{C}_{25} \mathrm{H}_{32} \mathrm{O}_{4} \mathrm{SiH}^{+}[\mathrm{M}+\mathrm{H}]^{+} 425.2148$, found 425.2138 .

\section{1-(5-acetoxy-2-hydroxy-4-methylphenyl)ethyl acetate (17)}<smiles>CC(=O)Oc1cc(C(C)O)c(O)cc1C</smiles>

3

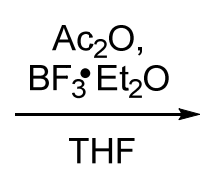

THF<smiles>CC(=O)Oc1cc(C(C)OC(C)=O)c(O)cc1C</smiles>

17

A solution of $\mathrm{BF}_{3} \cdot \mathrm{Et}_{2} \mathrm{O}(7.4 \mu \mathrm{L}, 0.06 \mathrm{mmol}, 0.25$ equiv $)$ in $\mathrm{Ac}_{2} \mathrm{O}(0.66 \mathrm{~mL})$ was added dropwise to a suspension of 4-hydroxy-5-(1-hydroxyethyl)-2-methylphenyl acetate (3) (50.0 $\mathrm{mg}, 0.24 \mathrm{mmol}, 1.0$ equiv) in THF with stirring under ice-cooling, then warmed to room temperature. After being stirred for $20 \mathrm{~h}$ under the same conditions, the reaction mixture was quenched with saturated $\mathrm{NaHCO}_{3}$ (aq.). The aqueous phase was extracted with ethyl acetate ( $3 \times 20 \mathrm{~mL})$. The combined organic phases were washed with brine $(50 \mathrm{~mL})$, dried over $\mathrm{Na}_{2} \mathrm{SO}_{4}$, and concentrated in vacuo. The residue was purified by column chromatography $(5: 1$ petroleum ether/ethyl acetate), provided compound $17(50.0 \mathrm{mg}, 82 \%)$ as colorless oil. ${ }^{1} \mathrm{H}$ $\operatorname{NMR}\left(400 \mathrm{MHz}, \mathrm{CDCl}_{3}\right) \delta 7.41(\mathrm{~s}, 1 \mathrm{H}), 6.97(\mathrm{~s}, 1 \mathrm{H}), 6.79(\mathrm{~s}, 1 \mathrm{H}), 5.96(\mathrm{q}, J=6.7 \mathrm{~Hz}, 1 \mathrm{H})$, $2.31(\mathrm{~s}, 3 \mathrm{H}), 2.11(\mathrm{~s}, 3 \mathrm{H}), 2.07(\mathrm{~s}, 3 \mathrm{H}), 1.60(\mathrm{~d}, J=6.7 \mathrm{~Hz}, 3 \mathrm{H}) .{ }^{13} \mathrm{C} \mathrm{NMR}\left(100 \mathrm{MHz}, \mathrm{CDCl}_{3}\right)$ 
$\delta 172.7,169.6,152.0,142.9,132.2,124.8,120.2,120.1,67.6,21.1,20.7,19.7,16.0$. IR (neat, $\left.\mathrm{cm}^{-1}\right): v 3423,2982,2933,1758,1739,1417,1371,1215,1052,918$. HRMS (ESIMS) calcd for $\mathrm{C}_{13} \mathrm{H}_{16} \mathrm{O}_{5} \mathrm{Na}^{+}[\mathrm{M}+\mathrm{Na}]^{+}$275.0895, found 275.0886.

\section{(5aS,10aR,11R)-7-hydroxy-5a-(hydroxymethyl)-3,8,11-trimethyl-5a,10a-dihydro-11H-be} nzofuro[3,2-b]chromen-2-yl acetate (18)<smiles>Cc1cc2occ(CO)c2cc1O</smiles>

Paeoveitol D<smiles>CC(=O)Oc1cc(C(C)OC(C)=O)c(O)cc1C</smiles>

17

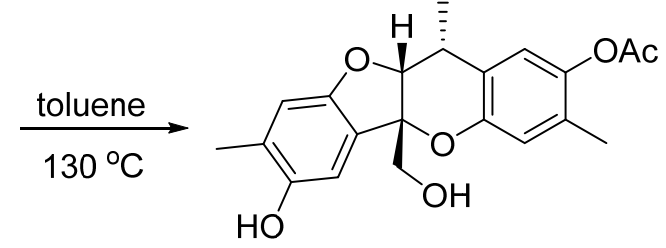

18

A sealed tube was charged with paeoveitol D (13.4 mg, $0.075 \mathrm{mmol}, 1.0$ equiv), 2-(1-hydroxyethyl)-5-methyl-4-((triethylsilyl)oxy) phenol (17) (22.7 mg, 0.09 mmol, 1.2 equiv), and toluene ( $1 \mathrm{~mL})$. The tube was filled with argon and was then sealed and heated at $130{ }^{\circ} \mathrm{C}$ for $48 \mathrm{~h}$ in a drying oven. The resulting reaction mixture was purified by column chromatography (2:1 petroleum ether/ethyl acetate) gave compound $18(15 \mathrm{mg}, 55 \%)$ as a white oil. ${ }^{1} \mathrm{H}$ NMR (400 MHz, $\left.\mathrm{CD}_{3} \mathrm{OD}\right) \delta 6.80(\mathrm{~s}, 1 \mathrm{H}), 6.71(\mathrm{~s}, 1 \mathrm{H}), 6.54(\mathrm{~s}, 1 \mathrm{H}), 6.32(\mathrm{~s}, 1 \mathrm{H})$, $5.03(\mathrm{~d}, J=3.0 \mathrm{~Hz}, 1 \mathrm{H}), 4.16(\mathrm{~d}, J=11.8 \mathrm{~Hz}, 1 \mathrm{H}), 3.91(\mathrm{~d}, J=11.8 \mathrm{~Hz}, 1 \mathrm{H}), 3.08(\mathrm{~m}, 1 \mathrm{H})$, 2.24 (s, 3H), 2.06 (s, 3H), 1.97 (s, 3H), 1.52 (d, J=7.0 Hz, 3H). ${ }^{13} \mathrm{C}$ NMR (100 MHz, CD 3 OD) $\delta 155.1,153.4,150.4,145.6,129.8,129.2,129.1,125.3,120.7,120.2,112.1,110.9,90.4,89.8$, 67.2, 33.7, 26.9, 20.6, 16.8, 15.9, 13.5. HRMS (ESIMS) calcd for $\mathrm{C}_{21} \mathrm{H}_{22} \mathrm{O}_{6} \mathrm{Na}^{+}[\mathrm{M}+\mathrm{Na}]^{+}$ 393.1314, found 393.1301.

\section{Paeoveitol}

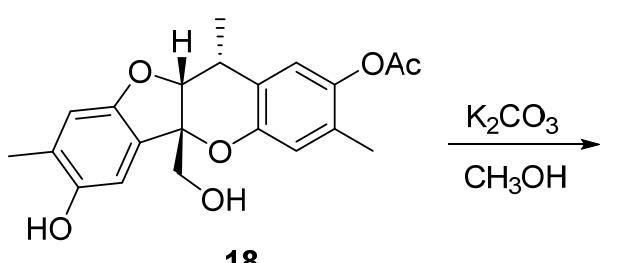

18

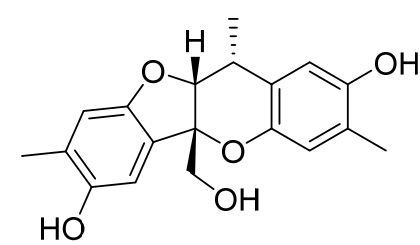

Paeoveitol

To a $5 \mathrm{~mL}$ round bottom flask equipped with a stir bar were added compound 18 (9.2 mg, 
0.025 mmol, 1.0 equiv) and $\mathrm{CH}_{3} \mathrm{OH}(1 \mathrm{~mL})$. The solution was stirred at room temperature, the anhydrous $\mathrm{K}_{2} \mathrm{CO}_{3}(3.5 \mathrm{mg}, 0.025 \mathrm{mmol}, 1.0$ equiv) was added. The progress of reaction was monitored by TLC. The reaction was quenched with saturated $\mathrm{NH}_{4} \mathrm{Cl}$ (aq.). The resulting mixture was extracted with ethyl acetate $(3 \times 20 \mathrm{~mL})$. The organic layer was washed with water followed by brine, and dried over anhydrous $\mathrm{Na}_{2} \mathrm{SO}_{4}$. It was filtered and concentrated in vacuo. The residue was purified by column chromatography (2:1 petroleum ether/ethyl acetate) provided the natural product Paeoveitol $(6.6 \mathrm{mg}, 80 \%)$ as a white foam, (m. p. 150 152 $\left.{ }^{\circ} \mathrm{C}\right) .{ }^{1} \mathrm{H}$ NMR (400 MHz, Pyr) $\delta 10.74$ (s, 1H), 10.68 (s, 1H), 7.46 (s, 1H), 7.11 (s, $1 \mathrm{H}), 6.88(\mathrm{~s}, 1 \mathrm{H}), 6.62(\mathrm{~s}, 1 \mathrm{H}), 5.54(\mathrm{~d}, J=3.0 \mathrm{~Hz}, 1 \mathrm{H}), 4.64(\mathrm{~d}, J=11.5 \mathrm{~Hz}, 1 \mathrm{H}), 4.45(\mathrm{~d}, J=$ $11.6 \mathrm{~Hz}, 1 \mathrm{H}), 3.41(\mathrm{~m}, 1 \mathrm{H}), 2.24(\mathrm{~s}, 3 \mathrm{H}), 2.20(\mathrm{~s}, 3 \mathrm{H}), 1.61(\mathrm{~d}, J=7.0 \mathrm{~Hz}, 3 \mathrm{H}) .{ }^{13} \mathrm{C} \mathrm{NMR}$ (100 MHz, Pyr) $\delta 155.0,152.4,151.3,148.0,128.6,128.4,126.5,123.7,120.8,113.6,112.4$, 111.5, 90.2, 89.9, 67.4, 33.6, 17.6, 16.8, 14.1. IR (neat, $\mathrm{cm}^{-1}$ ): v 3402, 2283, 2269, 2250, 1654, 1538, 1302, 965, 824, 533. HRMS (ESIMS) calcd for $\mathrm{C}_{19} \mathrm{H}_{20} \mathrm{O}_{5} \mathrm{Na}^{+}[\mathrm{M}+\mathrm{Na}]^{+} 351.1208$, found 351.1199 .

\section{References:}

(1) Macías, F. A.; Chinchilla, D.; Molinillo, J. M. G. Marín, D.; Varela, R. M.; Torres, A. Tetrahedron, 2003, 59, $1679-1683$.

(2) Bian, J. L.; Deng, B.; Xu, L. L.; Xu, X. L.; Wang, N.; Huc, T. H.; Yao, Z. Y.; Du, J. Y.; Yang, L.; Lei, Y. H.; Li, X.; Sun, H. P.; Zhang, X. J.; You, Q. D. Eur. J. Med. Chem. 2014, 82, 56-57. 


\section{X-ray crystallography of compound 5'}

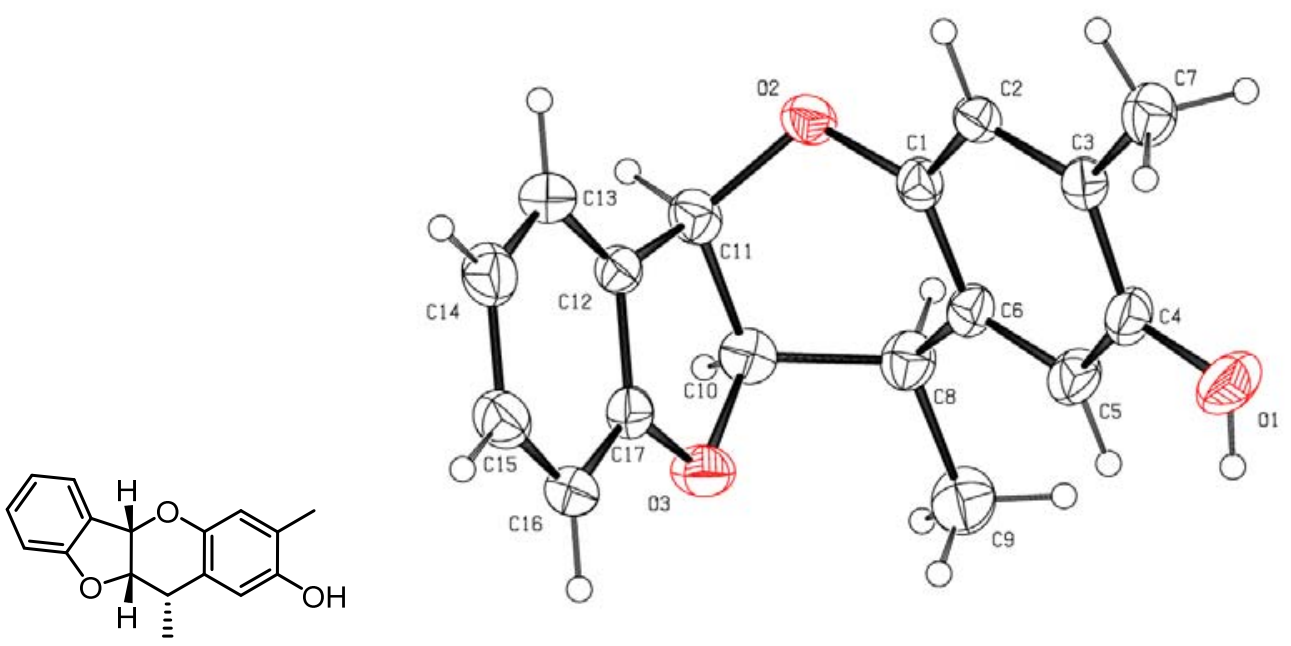

The crystallographic data of 5 ' were summarized in the following table.

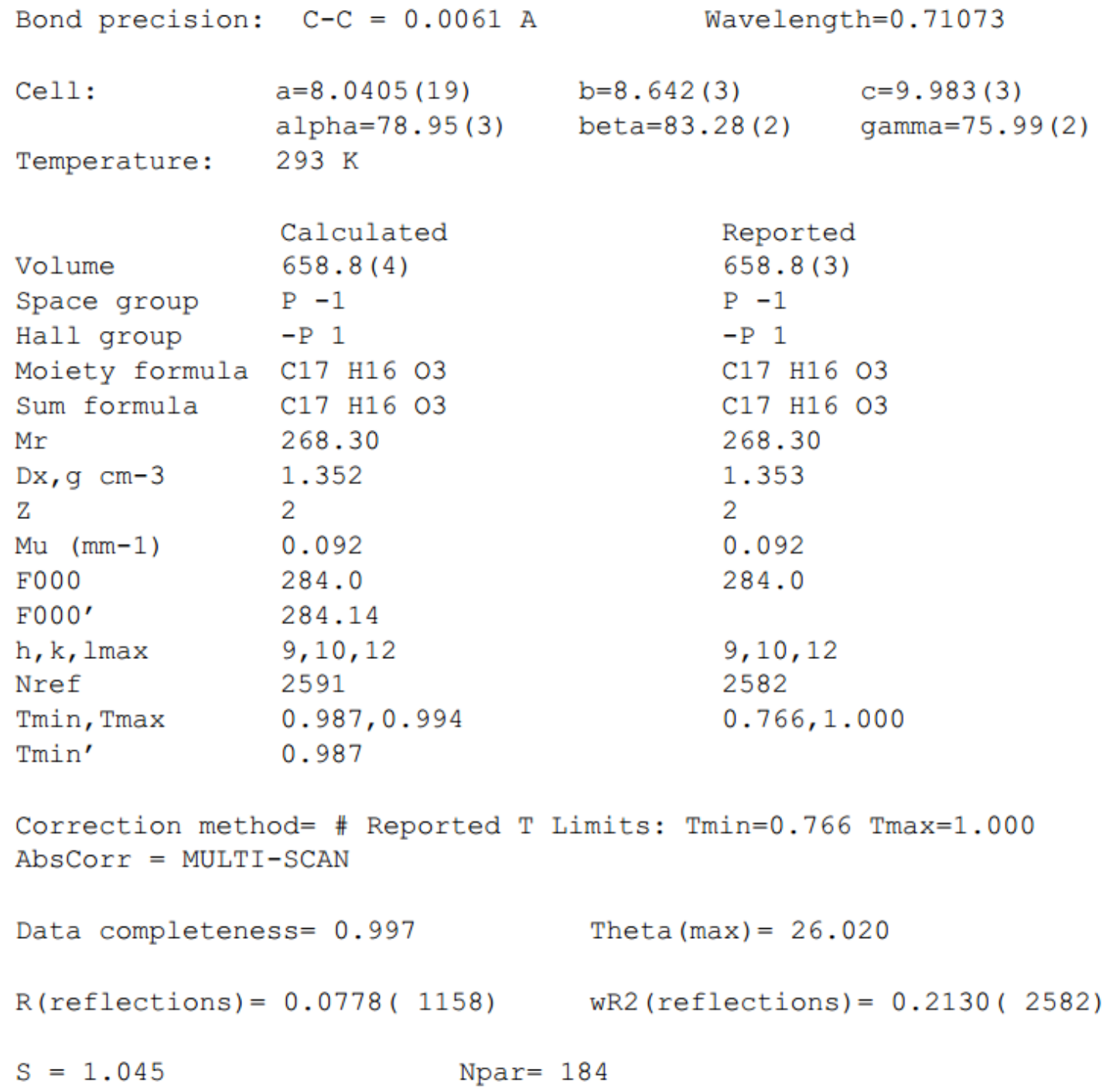


CIF file of $4 \mathrm{a}$ can be obtained from the Cambridge Crystallographic Data Centre using deposition number CCDC 1499563. Copies of the data can be obtained, free of charge, on application to the CCDC, 12 Union Road, Cambridge CB2 1EZ, UK [fax:+44(1223)336033; e-mail: deposit@ccdc.cam.ac.uk].

Table 1Crystal data and structure refinement for Xzx_0615.

$\begin{array}{cc}\text { Identification code } & \text { Xzx_0615 } \\ \text { Empirical formula } & \mathrm{C}_{17} \mathrm{H}_{16} \mathrm{O}_{3} \\ \text { Formula weight } & 268.30 \\ \text { Temperature/K } & 293(2) \\ \text { Crystal system } & \text { triclinic } \\ \text { Space group } & \mathrm{P}-1 \\ \mathrm{a} / \AA & 8.0405(19) \\ \mathrm{b} / \AA & 8.642(3) \\ \mathrm{c} / \AA & 9.983(3) \\ \alpha /{ }^{\circ} & 78.95(3) \\ \beta /{ }^{\circ} & 83.28(2) \\ \gamma /{ }^{\circ} & 75.99(2) \\ \mathrm{Volume} / \AA^{3} & 658.8(3) \\ \mathrm{Z} & 2 \\ \rho_{\text {calc }} / \mathrm{cm}^{3} & 1.353 \\ \mu / \mathrm{mm}^{-1} & 0.092 \\ \mathrm{~F}(000) & 284.0\end{array}$

Crystal size $/ \mathrm{mm}^{3} \quad 0.14 \times 0.12 \times 0.07$

Radiation $\operatorname{MoK} \alpha(\lambda=0.71073)$

$2 \Theta$ range for data collection $/{ }^{\circ}$

6.88 to 52.04

Index ranges $\quad-9 \leq \mathrm{h} \leq 9,-10 \leq \mathrm{k} \leq 10,-12 \leq 1 \leq 12$

Reflections collected

3967

Independent reflections

Data/restraints/parameters

Goodness-of-fit on $\mathrm{F}^{2}$ $2582\left[\mathrm{R}_{\text {int }}=0.0462, \mathrm{R}_{\text {sigma }}=0.1230\right]$

Final $\mathrm{R}$ indexes $[\mathrm{I}>=2 \sigma(\mathrm{I})]$

$2582 / 0 / 184$

\subsection{5}

Final $\mathrm{R}$ indexes [all data]

Largest diff. peak/hole / e $\AA^{-3}$

$$
\begin{aligned}
& \mathrm{R}_{1}=0.0778, \mathrm{wR}_{2}=0.1483 \\
& \mathrm{R}_{1}=0.1718, \mathrm{wR}_{2}=0.2130
\end{aligned}
$$

$0.23 /-0.19$ 
Table 2 Fractional Atomic Coordinates $\left(\times 10^{4}\right)$ and Equivalent Isotropic Displacement Parameters $\left(\AA^{2} \times 10^{3}\right)$ for $x z x \_0615$. $U_{\text {eq }}$ is defined as $1 / 3$ of of the trace of the orthogonalised $\mathrm{U}_{\mathrm{IJ}}$ tensor.

\begin{tabular}{lllll} 
Atom & \multicolumn{1}{c}{$\boldsymbol{x}$} & \multicolumn{1}{c}{$\mathbf{U}(\mathbf{e q})$} \\
$\mathrm{O} 1$ & $1938(5)$ & $13574(4)$ & $123(3)$ & $74.8(11)$ \\
O2 & $1109(3)$ & $7882(3)$ & $3436(3)$ & $47.0(8)$ \\
O3 & $5269(3)$ & $6797(4)$ & $1955(3)$ & $55.3(9)$ \\
C1 & $1255(5)$ & $9329(5)$ & $2569(4)$ & $40.7(10)$ \\
C2 & $581(4)$ & $10790(5)$ & $3001(4)$ & $39.1(10)$ \\
C3 & $754(5)$ & $12227(5)$ & $2165(4)$ & $40.9(10)$ \\
C4 & $1675(5)$ & $12142(5)$ & $903(4)$ & $49.6(12)$ \\
C5 & $2268(6)$ & $10662(5)$ & $458(4)$ & $51.5(12)$ \\
C6 & $2041(5)$ & $9227(5)$ & $1273(4)$ & $40.2(10)$ \\
C7 & $31(6)$ & $13849(5)$ & $2619(4)$ & $55.2(12)$ \\
C8 & $2548(6)$ & $7574(5)$ & $891(4)$ & $50.3(12)$ \\
C9 & $3437(6)$ & $7484(6)$ & $-525(4)$ & $67.7(15)$ \\
C10 & $3568(5)$ & $6445(5)$ & $2033(4)$ & $46.9(11)$ \\
C11 & $2716(5)$ & $6679(5)$ & $3483(4)$ & $45.8(11)$ \\
C12 & $4103(5)$ & $7112(5)$ & $4120(4)$ & $38.3(10)$ \\
C13 & $4141(5)$ & $7471(5)$ & $5406(4)$ & $50.8(12)$ \\
C14 & $5594(6)$ & $7878(6)$ & $5709(4)$ & $60.0(14)$ \\
C15 & $6973(6)$ & $7934(6)$ & $4752(5)$ & $56.4(13)$ \\
C16 & $6944(5)$ & $7573(5)$ & $3477(4)$ & $50.1(12)$ \\
C17 & $5473(5)$ & $7179(5)$ & $3191(4)$ & $42.1(11)$
\end{tabular}


Table 3 Anisotropic Displacement Parameters $\left(\AA^{2} \times 10^{3}\right)$ for xzx_0615. The Anisotropic displacement factor exponent takes the form: $-2 \pi^{2}\left[h^{2} a^{* 2} U_{11}+2 h k a * b * U_{12}+\ldots\right]$.

\begin{tabular}{lllllll} 
Atom & \multicolumn{1}{c}{$\mathbf{U}_{\mathbf{1 1}}$} & \multicolumn{1}{c}{$\mathbf{U}_{\mathbf{2 2}}$} & \multicolumn{1}{c}{$\mathbf{U}_{\mathbf{3 3}}$} & \multicolumn{1}{c}{$\mathbf{U}_{23}$} & \multicolumn{1}{c}{$\mathbf{U}_{\mathbf{1 3}}$} & \multicolumn{1}{c}{$\mathbf{U}_{\mathbf{1 2}}$} \\
$\mathrm{O} 1$ & $100(3)$ & $48(2)$ & $58(2)$ & $6.6(18)$ & $20.6(19)$ & $-4.8(19)$ \\
$\mathrm{O} 2$ & $37.8(15)$ & $41.1(18)$ & $58.1(18)$ & $-2.7(15)$ & $3.1(13)$ & $-8.6(13)$ \\
$\mathrm{O} 3$ & $45.3(18)$ & $75(2)$ & $48.1(18)$ & $-18.3(17)$ & $2.9(14)$ & $-14.2(16)$ \\
C1 & $36(2)$ & $41(3)$ & $45(2)$ & $1(2)$ & $-9.5(19)$ & $-11.2(19)$ \\
C2 & $34(2)$ & $44(3)$ & $36(2)$ & $-8(2)$ & $-2.2(18)$ & $-2.5(19)$ \\
C3 & $41(2)$ & $39(3)$ & $40(2)$ & $-3(2)$ & $-6.4(19)$ & $-4.3(19)$ \\
C4 & $60(3)$ & $40(3)$ & $41(2)$ & $3(2)$ & $-4(2)$ & $-3(2)$ \\
C5 & $63(3)$ & $51(3)$ & $34(2)$ & $-8(2)$ & $0(2)$ & $-3(2)$ \\
C6 & $44(2)$ & $43(3)$ & $34(2)$ & $-3(2)$ & $-7.2(19)$ & $-11(2)$ \\
C7 & $63(3)$ & $43(3)$ & $57(3)$ & $-3(2)$ & $-8(2)$ & $-8(2)$ \\
C8 & $57(3)$ & $49(3)$ & $46(3)$ & $-9(2)$ & $-8(2)$ & $-11(2)$ \\
C9 & $81(4)$ & $72(4)$ & $49(3)$ & $-23(3)$ & $-7(3)$ & $-2(3)$ \\
C10 & $49(3)$ & $42(3)$ & $53(3)$ & $-13(2)$ & $-2(2)$ & $-12(2)$ \\
C11 & $45(2)$ & $34(3)$ & $56(3)$ & $-6(2)$ & $-3(2)$ & $-5.3(19)$ \\
C12 & $35(2)$ & $35(3)$ & $42(2)$ & $-1(2)$ & $-2.4(19)$ & $-8.1(18)$ \\
C13 & $45(3)$ & $56(3)$ & $47(3)$ & $-8(2)$ & $4(2)$ & $-8(2)$ \\
C14 & $61(3)$ & $64(4)$ & $50(3)$ & $-13(3)$ & $-11(2)$ & $3(3)$ \\
C15 & $44(3)$ & $58(3)$ & $65(3)$ & $-6(3)$ & $-7(2)$ & $-10(2)$ \\
C16 & $38(2)$ & $55(3)$ & $52(3)$ & $-2(2)$ & $1(2)$ & $-7(2)$ \\
C17 & $43(2)$ & $39(3)$ & $40(2)$ & $-2(2)$ & $-5(2)$ & $-4(2)$ \\
& & & & & &
\end{tabular}


Table 4 Bond Lengths for xzx_0615.

\begin{tabular}{lllllll}
\multicolumn{3}{c}{ Atom Atom Length/ $\AA$} & \multicolumn{2}{c}{ Atom Atom } & $\begin{array}{c}\text { Length/ } \\
\AA\end{array}$ \\
$\mathrm{O} 1$ & $\mathrm{C} 4$ & $1.379(5)$ & $\mathrm{C} 6$ & $\mathrm{C} 8$ & $1.498(6)$ \\
$\mathrm{O} 2$ & $\mathrm{C} 1$ & $1.400(5)$ & $\mathrm{C} 8$ & $\mathrm{C} 9$ & $1.514(5)$ \\
$\mathrm{O} 2$ & $\mathrm{C} 11$ & $1.449(4)$ & $\mathrm{C} 8$ & $\mathrm{C} 10$ & $1.527(5)$ \\
$\mathrm{O} 3$ & $\mathrm{C} 10$ & $1.462(5)$ & $\mathrm{C} 10$ & $\mathrm{C} 11$ & $1.555(5)$ \\
$\mathrm{O} 3$ & $\mathrm{C} 17$ & $1.375(4)$ & $\mathrm{C} 11$ & $\mathrm{C} 12$ & $1.499(5)$ \\
$\mathrm{C} 1$ & $\mathrm{C} 2$ & $1.377(5)$ & $\mathrm{C} 12$ & $\mathrm{C} 13$ & $1.383(5)$ \\
$\mathrm{C} 1$ & $\mathrm{C} 6$ & $1.380(5)$ & $\mathrm{C} 12$ & $\mathrm{C} 17$ & $1.359(5)$ \\
$\mathrm{C} 2$ & $\mathrm{C} 3$ & $1.385(5)$ & $\mathrm{C} 13$ & $\mathrm{C} 14$ & $1.379(6)$ \\
$\mathrm{C} 3$ & $\mathrm{C} 4$ & $1.391(5)$ & $\mathrm{C} 14$ & $\mathrm{C} 15$ & $1.380(6)$ \\
$\mathrm{C} 3$ & $\mathrm{C} 7$ & $1.514(5)$ & $\mathrm{C} 15$ & $\mathrm{C} 16$ & $1.371(5)$ \\
$\mathrm{C} 4$ & $\mathrm{C} 5$ & $1.390(6)$ & $\mathrm{C} 16$ & $\mathrm{C} 17$ & $1.381(6)$ \\
$\mathrm{C} 5$ & $\mathrm{C} 6$ & $1.386(6)$ & & &
\end{tabular}

Table 5 Bond Angles for xzx_0615.

\begin{tabular}{|c|c|c|c|c|c|c|c|}
\hline Atom & Atom & Atom & Angle $/^{\circ}$ & Atom & Atom & Atom & Angle $/^{\circ}$ \\
\hline $\mathrm{C} 1$ & $\mathrm{O} 2$ & C11 & $112.4(3)$ & C9 & C8 & $\mathrm{C} 10$ & $113.6(3)$ \\
\hline $\mathrm{C} 17$ & $\mathrm{O} 3$ & $\mathrm{C} 10$ & 107.7 & $\mathrm{O} 3$ & $\mathrm{C} 10$ & $\mathrm{C} 8$ & $109.1(4)$ \\
\hline $\mathrm{C} 2$ & $\mathrm{C} 1$ & $\mathrm{O} 2$ & $120.1(4)$ & $\mathrm{O} 3$ & $\mathrm{C} 10$ & $\mathrm{C} 11$ & $106.7(3)$ \\
\hline $\mathrm{C} 2$ & $\mathrm{C} 1$ & C6 & $122.2(4)$ & $\mathrm{C} 8$ & $\mathrm{C} 10$ & $\mathrm{C} 11$ & $112.7(3)$ \\
\hline 6 & $\mathrm{C} 1$ & $\mathrm{O} 2$ & 117.6 & $\mathrm{O} 2$ & C11 & $\mathrm{C} 10$ & $112.5(3)$ \\
\hline 1 & $\mathrm{C} 2$ & $\mathrm{C} 3$ & $120.7(4)$ & $\mathrm{O} 2$ & $\mathrm{C} 11$ & $\mathrm{C} 12$ & $114.4(3)$ \\
\hline 2 & $\mathrm{C} 3$ & $\mathrm{C} 4$ & $117.9(4)$ & $\mathrm{C} 12$ & C11 & $\mathrm{C} 10$ & $102.2(3)$ \\
\hline 2 & $\mathrm{C} 3$ & C7 & $121.7(4)$ & $\mathrm{C} 13$ & $\mathrm{C} 12$ & $\mathrm{C} 11$ & $130.7(4)$ \\
\hline $\mathrm{C4}$ & $\mathrm{C} 3$ & $\mathrm{C} 7$ & $120.3(4)$ & C17 & $\mathrm{C} 12$ & $\mathrm{C} 11$ & $109.4(3)$ \\
\hline 1 & $\mathrm{C} 4$ & $\mathrm{C} 3$ & $117.3(4)$ & C17 & $\mathrm{C} 12$ & $\mathrm{C} 13$ & $119.8(4)$ \\
\hline D1 & C4 & C5 & 122.4 & C14 & $\mathrm{C} 13$ & $\mathrm{C} 12$ & $118.3(4)$ \\
\hline $\mathrm{C} 5$ & $\mathrm{C} 4$ & $\mathrm{C} 3$ & $120.3(4)$ & $\mathrm{C} 13$ & $\mathrm{C} 14$ & $\mathrm{C} 15$ & $120.9(4)$ \\
\hline 6 & $\mathrm{C} 5$ & $\mathrm{C} 4$ & $121.7(4)$ & C16 & $\mathrm{C} 15$ & C14 & $121.0(5)$ \\
\hline $\mathrm{C} 1$ & C6 & $\mathrm{C} 5$ & $116.8(4)$ & $\mathrm{C} 15$ & C16 & $\mathrm{C} 17$ & $117.2(4)$ \\
\hline $\mathrm{C} 1$ & C6 & $\mathrm{C} 8$ & $116.5(4)$ & $\mathrm{O} 3$ & $\mathrm{C} 17$ & C16 & $123.3(4)$ \\
\hline 5 & C6 & C8 & $126.7(4)$ & $\mathrm{C} 12$ & $\mathrm{C} 17$ & $\mathrm{O} 3$ & $113.9(4)$ \\
\hline C6 & $\mathrm{C} 8$ & C9 & $115.6(4)$ & $\mathrm{C} 12$ & $\mathrm{C} 17$ & $\mathrm{C} 16$ & $122.8(4)$ \\
\hline 6 & $\mathrm{C} 8$ & C10 & $107.5(3)$ & & & & \\
\hline
\end{tabular}


Table 6 Torsion Angles for xzx_0615.

\begin{tabular}{llllllllll}
$\mathbf{A}$ & $\mathbf{B}$ & $\mathbf{C}$ & $\mathbf{D}$ & \multicolumn{1}{c}{ Angle $^{\circ}$} & \multicolumn{1}{|c}{$\mathbf{A}$} & $\mathbf{B}$ & $\mathbf{C}$ & \multicolumn{1}{c}{$\mathbf{D}$} & \multicolumn{1}{|c}{ Angle $/^{\circ}$} \\
$\mathrm{O} 1$ & $\mathrm{C} 4$ & $\mathrm{C} 5$ & $\mathrm{C} 6$ & $177.9(4)$ & $\mathrm{C} 7$ & $\mathrm{C} 3$ & $\mathrm{C} 4$ & $\mathrm{O} 1$ & $2.1(6)$ \\
$\mathrm{O} 2$ & $\mathrm{C} 1$ & $\mathrm{C} 2$ & $\mathrm{C} 3$ & $178.4(3)$ & $\mathrm{C} 7$ & $\mathrm{C} 3$ & $\mathrm{C} 4$ & $\mathrm{C} 5$ & $-176.9(4)$ \\
$\mathrm{O} 2$ & $\mathrm{C} 1$ & $\mathrm{C} 6$ & $\mathrm{C} 5$ & $-176.2(3)$ & $\mathrm{C} 8$ & $\mathrm{C} 10$ & $\mathrm{C} 11$ & $\mathrm{O} 2$ & $1.9(5)$ \\
$\mathrm{O} 2$ & $\mathrm{C} 1$ & $\mathrm{C} 6$ & $\mathrm{C} 8$ & $4.3(5)$ & $\mathrm{C} 8$ & $\mathrm{C} 10$ & $\mathrm{C} 11$ & $\mathrm{C} 12$ & $-121.3(4)$ \\
$\mathrm{O} 2$ & $\mathrm{C} 11$ & $\mathrm{C} 12$ & $\mathrm{C} 13$ & $58.0(6)$ & $\mathrm{C} 9$ & $\mathrm{C} 8$ & $\mathrm{C} 10$ & $\mathrm{O} 3$ & $56.1(5)$ \\
$\mathrm{O} 2$ & $\mathrm{C} 11$ & $\mathrm{C} 12$ & $\mathrm{C} 17$ & $-119.8(4)$ & $\mathrm{C} 9$ & $\mathrm{C} 8$ & $\mathrm{C} 10$ & $\mathrm{C} 11$ & $174.4(4)$ \\
$\mathrm{O} 3$ & $\mathrm{C} 10$ & $\mathrm{C} 11$ & $\mathrm{O} 2$ & $121.5(4)$ & $\mathrm{C} 10$ & $\mathrm{O} 3$ & $\mathrm{C} 17$ & $\mathrm{C} 12$ & $0.7(5)$ \\
$\mathrm{O} 3$ & $\mathrm{C} 10$ & $\mathrm{C} 11$ & $\mathrm{C} 12$ & $-1.6(4)$ & $\mathrm{C} 10$ & $\mathrm{O} 3$ & $\mathrm{C} 17$ & $\mathrm{C} 16$ & $179.7(4)$ \\
$\mathrm{C} 1$ & $\mathrm{O} 2$ & $\mathrm{C} 11$ & $\mathrm{C} 10$ & $-49.9(4)$ & $\mathrm{C} 10$ & $\mathrm{C} 11$ & $\mathrm{C} 12$ & $\mathrm{C} 13$ & $179.9(4)$ \\
$\mathrm{C} 1$ & $\mathrm{O} 2$ & $\mathrm{C} 11$ & $\mathrm{C} 12$ & $66.2(4)$ & $\mathrm{C} 10$ & $\mathrm{C} 11$ & $\mathrm{C} 12$ & $\mathrm{C} 17$ & $2.1(4)$ \\
$\mathrm{C} 1$ & $\mathrm{C} 2$ & $\mathrm{C} 3$ & $\mathrm{C} 4$ & $-2.1(6)$ & $\mathrm{C} 11$ & $\mathrm{O} 2$ & $\mathrm{C} 1$ & $\mathrm{C} 2$ & $-133.2(4)$ \\
$\mathrm{C} 1$ & $\mathrm{C} 2$ & $\mathrm{C} 3$ & $\mathrm{C} 7$ & $-179.7(3)$ & $\mathrm{C} 11$ & $\mathrm{O} 2$ & $\mathrm{C} 1$ & $\mathrm{C} 6$ & $48.8(4)$ \\
$\mathrm{C} 1$ & $\mathrm{C} 6$ & $\mathrm{C} 8$ & $\mathrm{C} 9$ & $-178.9(4)$ & $\mathrm{C} 11$ & $\mathrm{C} 12$ & $\mathrm{C} 13$ & $\mathrm{C} 14$ & $-178.1(4)$ \\
$\mathrm{C} 1$ & $\mathrm{C} 6$ & $\mathrm{C} 8$ & $\mathrm{C} 10$ & $-50.7(5)$ & $\mathrm{C} 11$ & $\mathrm{C} 12$ & $\mathrm{C} 17$ & $\mathrm{O} 3$ & $-1.8(5)$ \\
$\mathrm{C} 2$ & $\mathrm{C} 1$ & $\mathrm{C} 6$ & $\mathrm{C} 5$ & $5.8(6)$ & $\mathrm{C} 11$ & $\mathrm{C} 12$ & $\mathrm{C} 17$ & $\mathrm{C} 16$ & $179.1(4)$ \\
$\mathrm{C} 2$ & $\mathrm{C} 1$ & $\mathrm{C} 6$ & $\mathrm{C} 8$ & $-173.7(3)$ & $\mathrm{C} 12$ & $\mathrm{C} 13$ & $\mathrm{C} 14$ & $\mathrm{C} 15$ & $0.3(7)$ \\
$\mathrm{C} 2$ & $\mathrm{C} 3$ & $\mathrm{C} 4$ & $\mathrm{O} 1$ & $-175.6(4)$ & $\mathrm{C} 13$ & $\mathrm{C} 12$ & $\mathrm{C} 17$ & $\mathrm{O} 3$ & $-179.9(4)$ \\
$\mathrm{C} 2$ & $\mathrm{C} 3$ & $\mathrm{C} 4$ & $\mathrm{C} 5$ & $5.4(6)$ & $\mathrm{C} 13$ & $\mathrm{C} 12$ & $\mathrm{C} 17$ & $\mathrm{C} 16$ & $1.0(6)$ \\
$\mathrm{C} 3$ & $\mathrm{C} 4$ & $\mathrm{C} 5$ & $\mathrm{C} 6$ & $-3.2(7)$ & $\mathrm{C} 13$ & $\mathrm{C} 14$ & $\mathrm{C} 15$ & $\mathrm{C} 16$ & $-0.5(7)$ \\
$\mathrm{C} 4$ & $\mathrm{C} 5$ & $\mathrm{C} 6$ & $\mathrm{C} 1$ & $-2.4(6)$ & $\mathrm{C} 14$ & $\mathrm{C} 15$ & $\mathrm{C} 16$ & $\mathrm{C} 17$ & $1.0(7)$ \\
$\mathrm{C} 4$ & $\mathrm{C} 5$ & $\mathrm{C} 6$ & $\mathrm{C} 8$ & $177.1(4)$ & $\mathrm{C} 15$ & $\mathrm{C} 16$ & $\mathrm{C} 17$ & $\mathrm{O} 3$ & $179.8(4)$ \\
$\mathrm{C} 5$ & $\mathrm{C} 6$ & $\mathrm{C} 8$ & $\mathrm{C} 9$ & $1.6(6)$ & $\mathrm{C} 15$ & $\mathrm{C} 16$ & $\mathrm{C} 17$ & $\mathrm{C} 12$ & $-1.2(6)$ \\
$\mathrm{C} 5$ & $\mathrm{C} 6$ & $\mathrm{C} 8$ & $\mathrm{C} 10$ & $129.8(4)$ & $\mathrm{C} 17$ & $\mathrm{O} 3$ & $\mathrm{C} 10$ & $\mathrm{C} 8$ & $122.7(3)$ \\
$\mathrm{C} 6$ & $\mathrm{C} 1$ & $\mathrm{C} 2$ & $\mathrm{C} 3$ & $-3.7(6)$ & $\mathrm{C} 17$ & $\mathrm{O} 3$ & $\mathrm{C} 10$ & $\mathrm{C} 11$ & $0.7(4)$ \\
$\mathrm{C} 6$ & $\mathrm{C} 8$ & $\mathrm{C} 10$ & $\mathrm{O} 3$ & $-73.2(4)$ & $\mathrm{C} 17$ & $\mathrm{C} 12$ & $\mathrm{C} 13$ & $\mathrm{C} 14$ & $-0.5(6)$ \\
$\mathrm{C} 6$ & $\mathrm{C} 8$ & $\mathrm{C} 10$ & $\mathrm{C} 11$ & $45.1(5)$ & & & & & \\
& & & & & & & & &
\end{tabular}


Table 7 Hydrogen Atom Coordinates $\left(\AA \times 10^{4}\right)$ and Isotropic Displacement Parameters $\left(\AA^{2} \times 10^{3}\right)$ for xzx_0615.

\begin{tabular}{llllll} 
Atom & \multicolumn{1}{c}{$\boldsymbol{x}$} & \multicolumn{2}{c}{$\boldsymbol{z}$} & $\mathbf{U}(\mathbf{e q})$ \\
H1 & 2686 & 13377 & -493 & 112 \\
H2 & 2 & 10811 & 3862 & 47 \\
H5 & 2832 & 10634 & -408 & 62 \\
H7A & -412 & 13681 & 3558 & 83 \\
H7B & -878 & 14460 & 2058 & 83 \\
H7C & 924 & 14433 & 2528 & 83 \\
H8 & 1481 & 7211 & 906 & 60 \\
H9A & 2720 & 8200 & -1195 & 102 \\
H9B & 3641 & 6396 & -697 & 102 \\
H9C & 4512 & 7797 & -579 & 102 \\
H10 & 3691 & 5319 & 1923 & 56 \\
H11 & 2507 & 5644 & 3984 & 55 \\
H13 & 3210 & 7439 & 6050 & 61 \\
H14 & 5644 & 8119 & 6570 & 72 \\
H15 & 7936 & 8220 & 4975 & 68 \\
H16 & 7876 & 7592 & 2833 & 60
\end{tabular}




\section{5. ${ }^{1} \mathrm{H}$ and ${ }^{13} \mathrm{C}$ NMR spectra of new compounds}

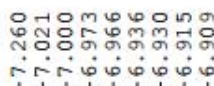

ririo í<smiles>CC(=O)Oc1ccc(OC(C)=O)c(C)c1</smiles>

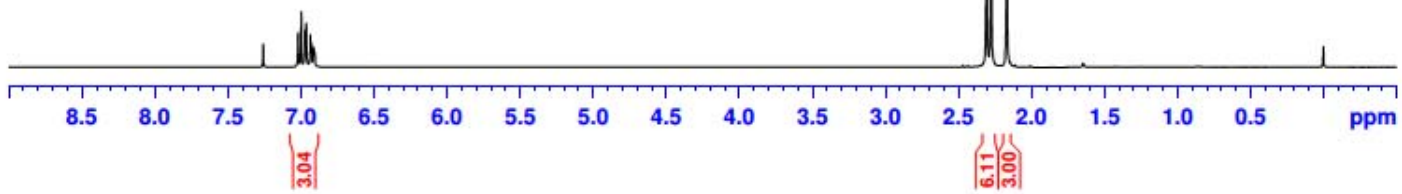<smiles>CC(=O)Oc1ccc(OC(C)=O)c(C)c1</smiles>

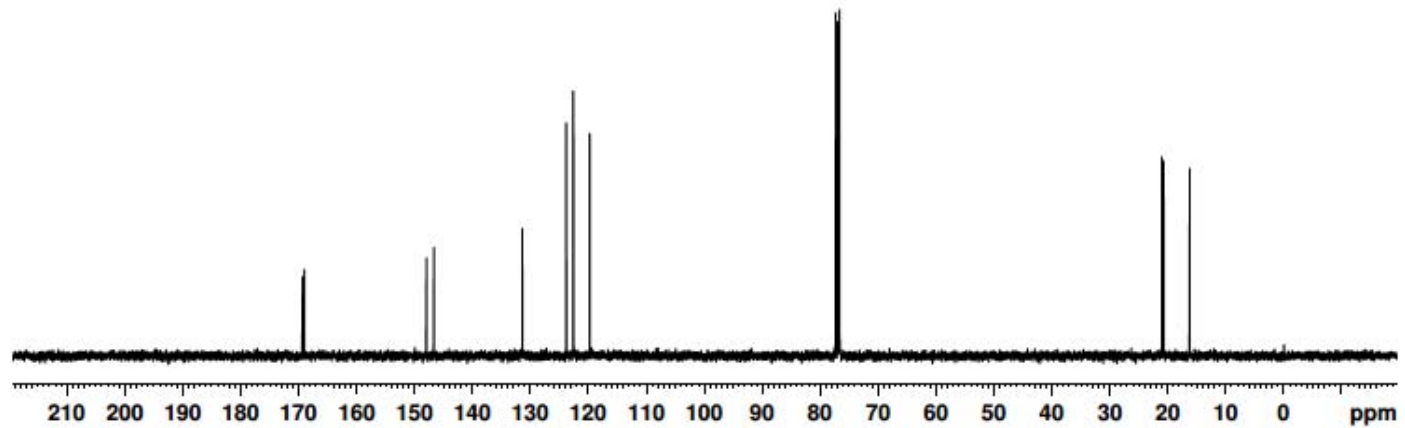




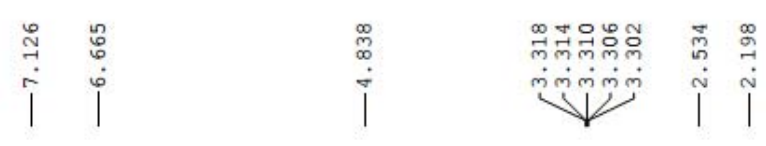<smiles>CC(=O)c1cc(O)c(C)cc1O</smiles>
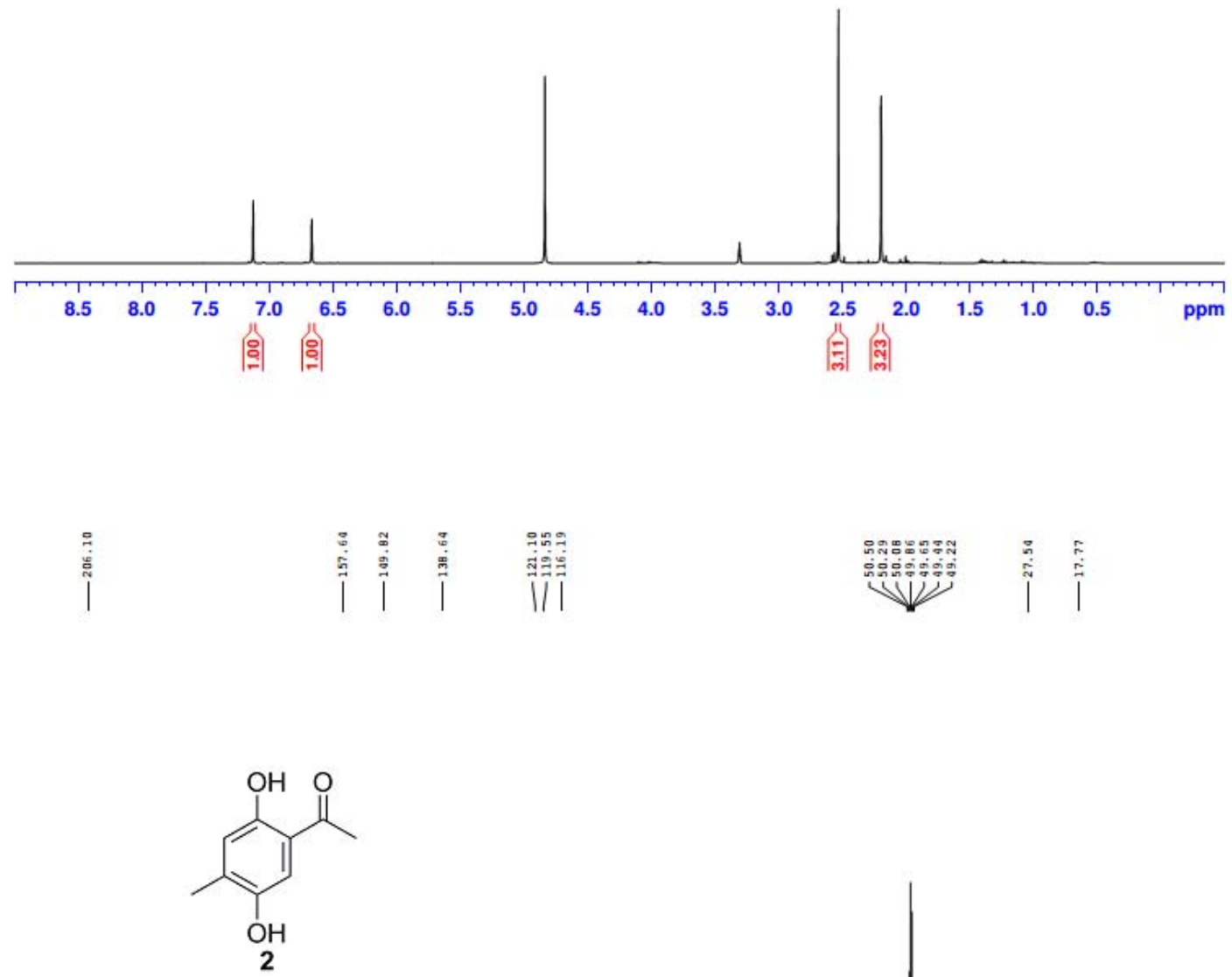

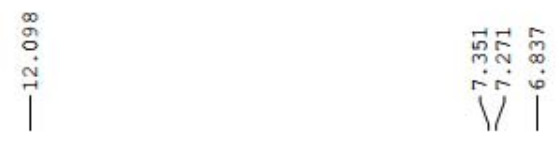

주유ำ<smiles>CC(=O)Oc1cc(C(C)=O)c(O)cc1C</smiles>

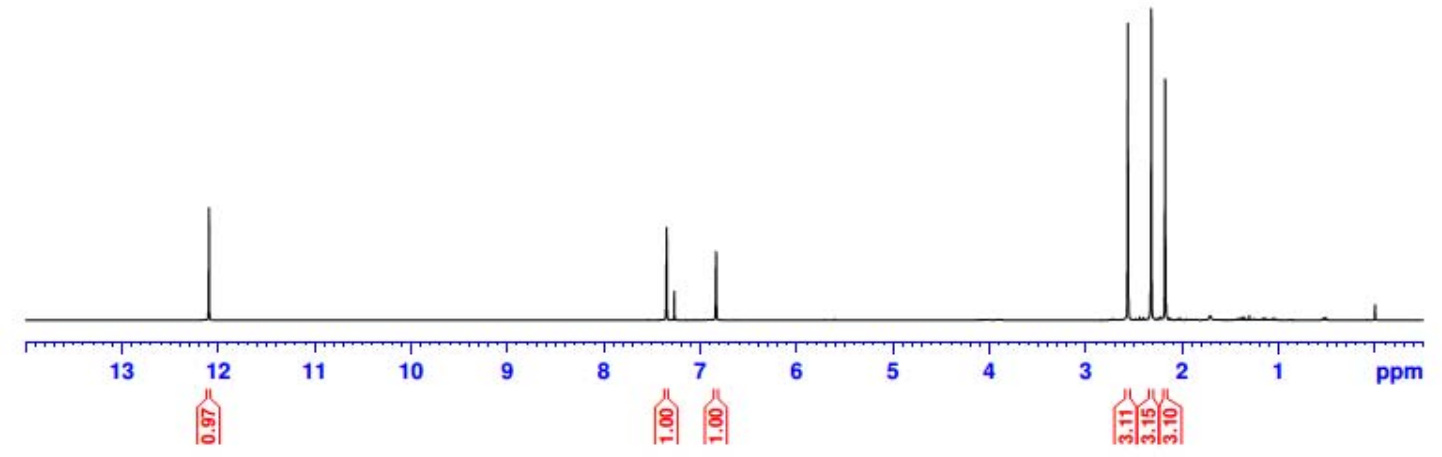

|

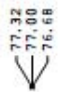

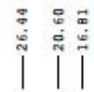<smiles>CC(=O)Oc1cc(C(C)=O)c(O)cc1C</smiles>

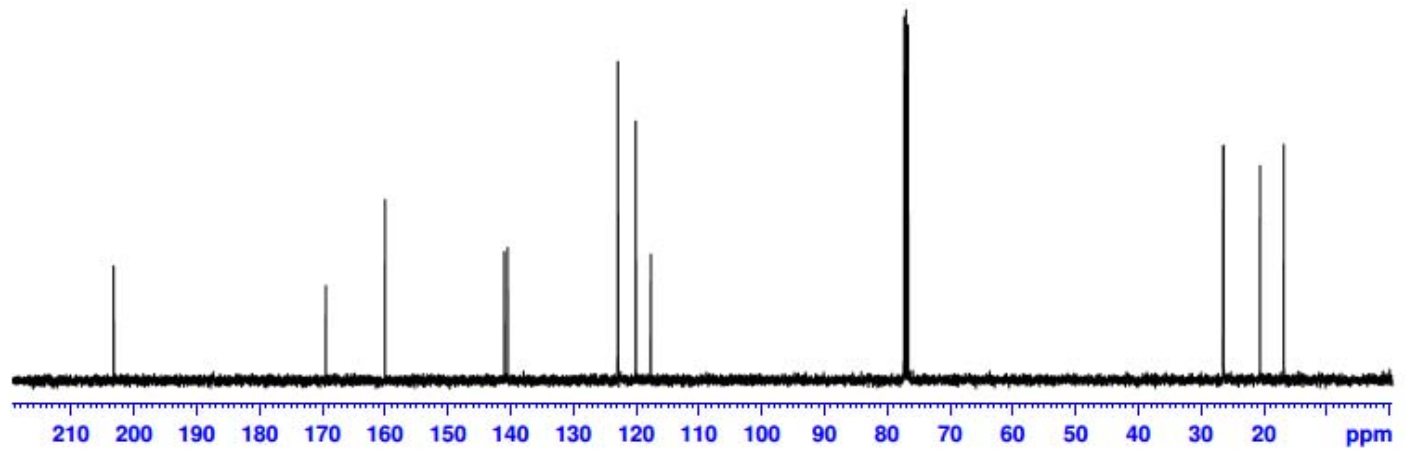



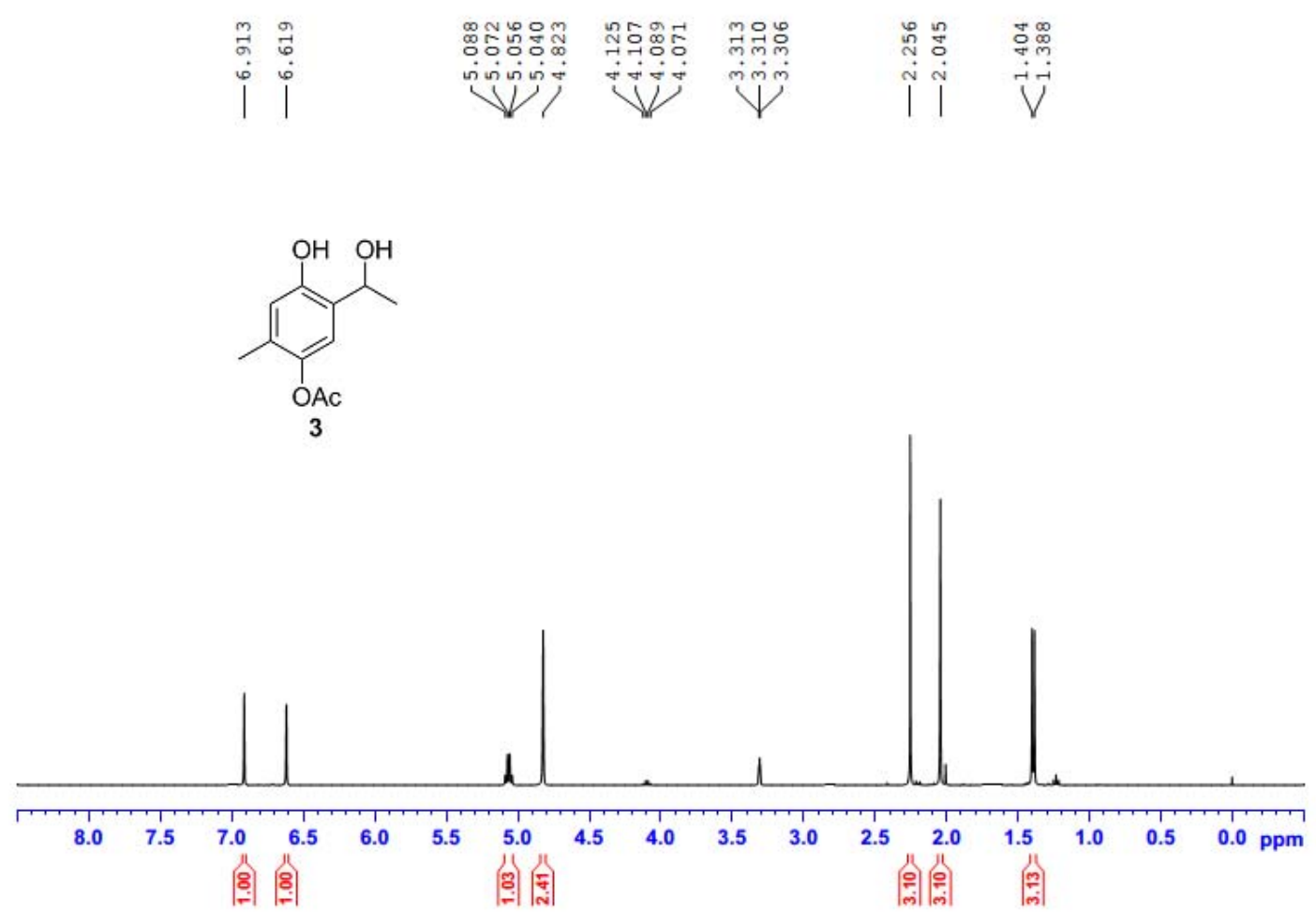

†
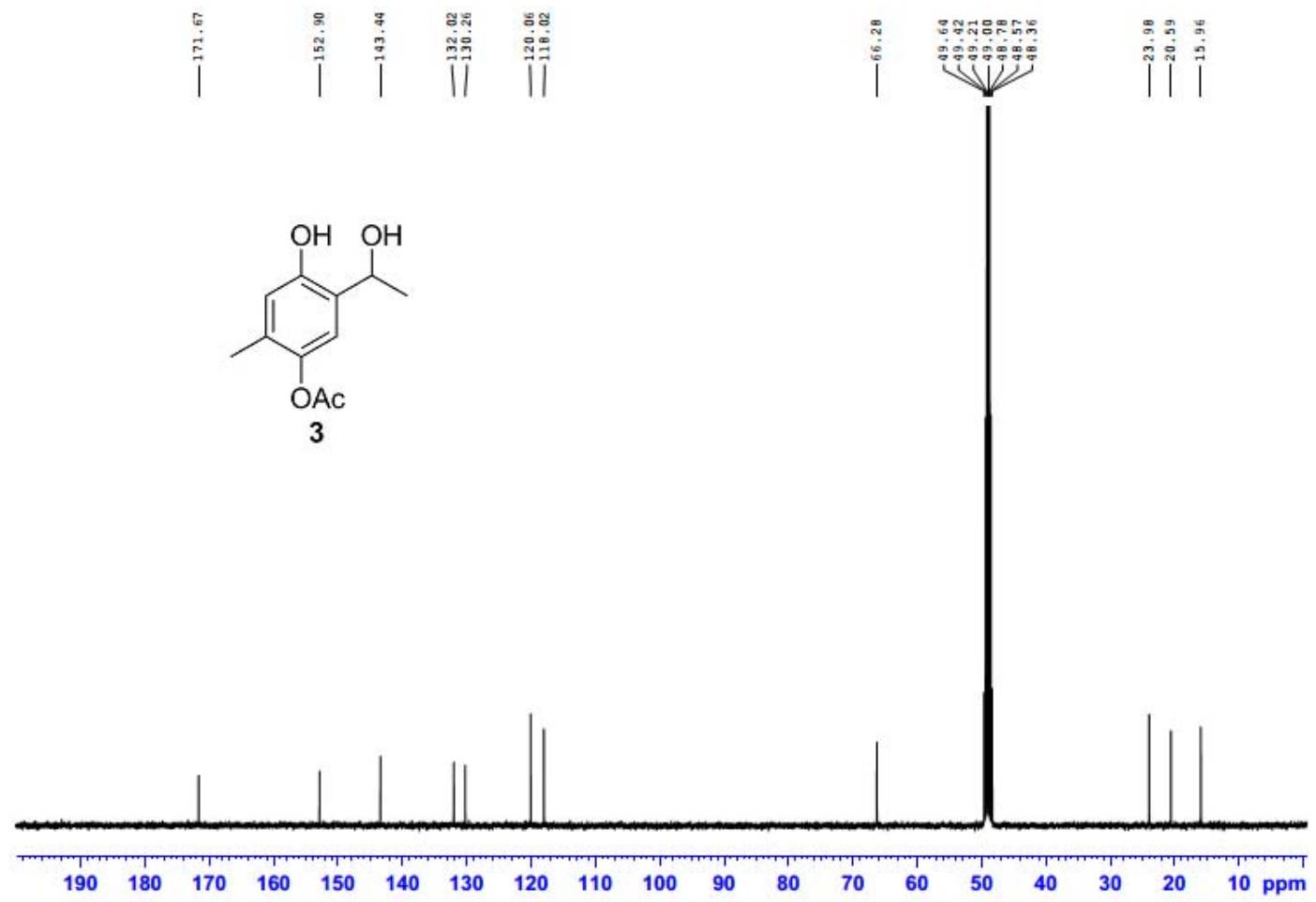

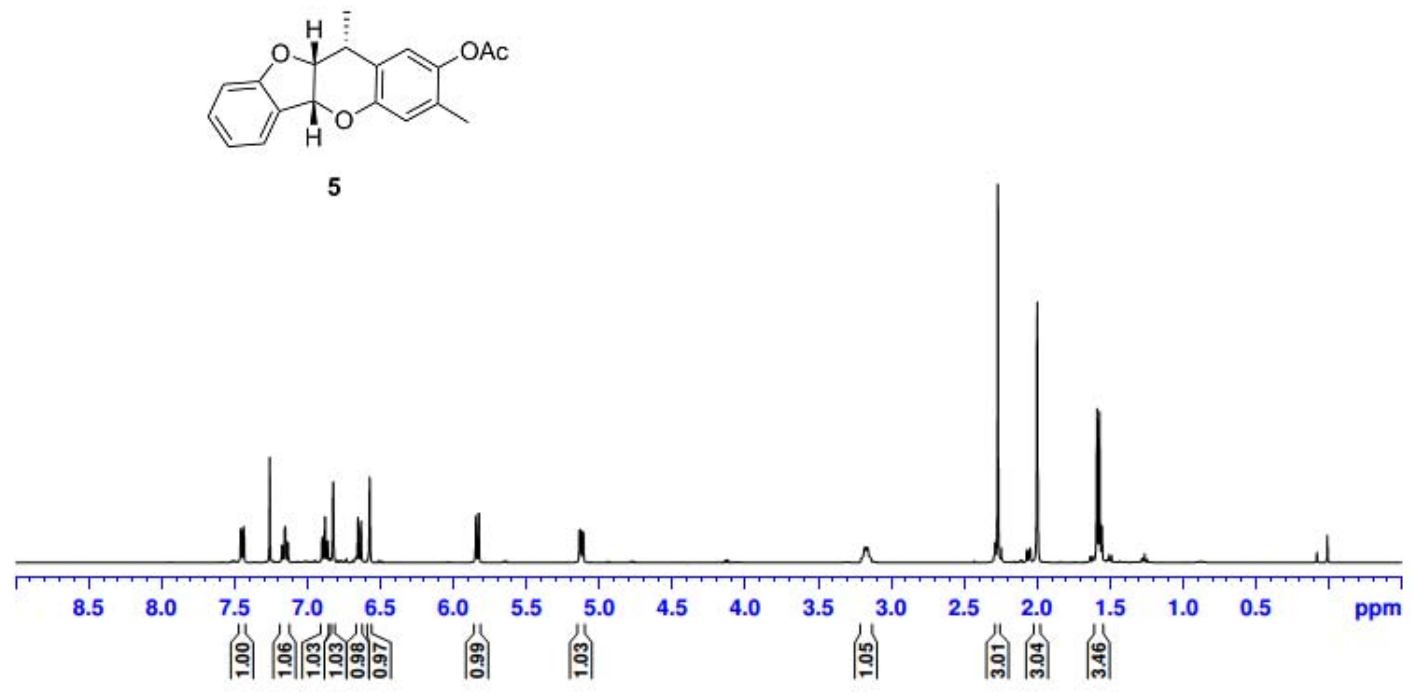

|

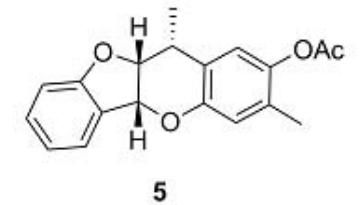

5

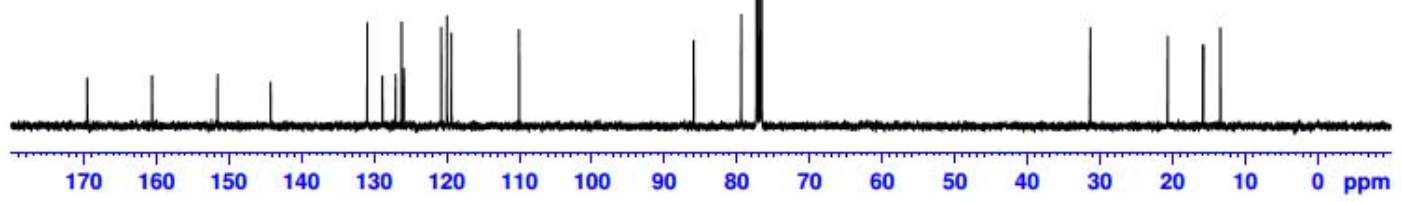




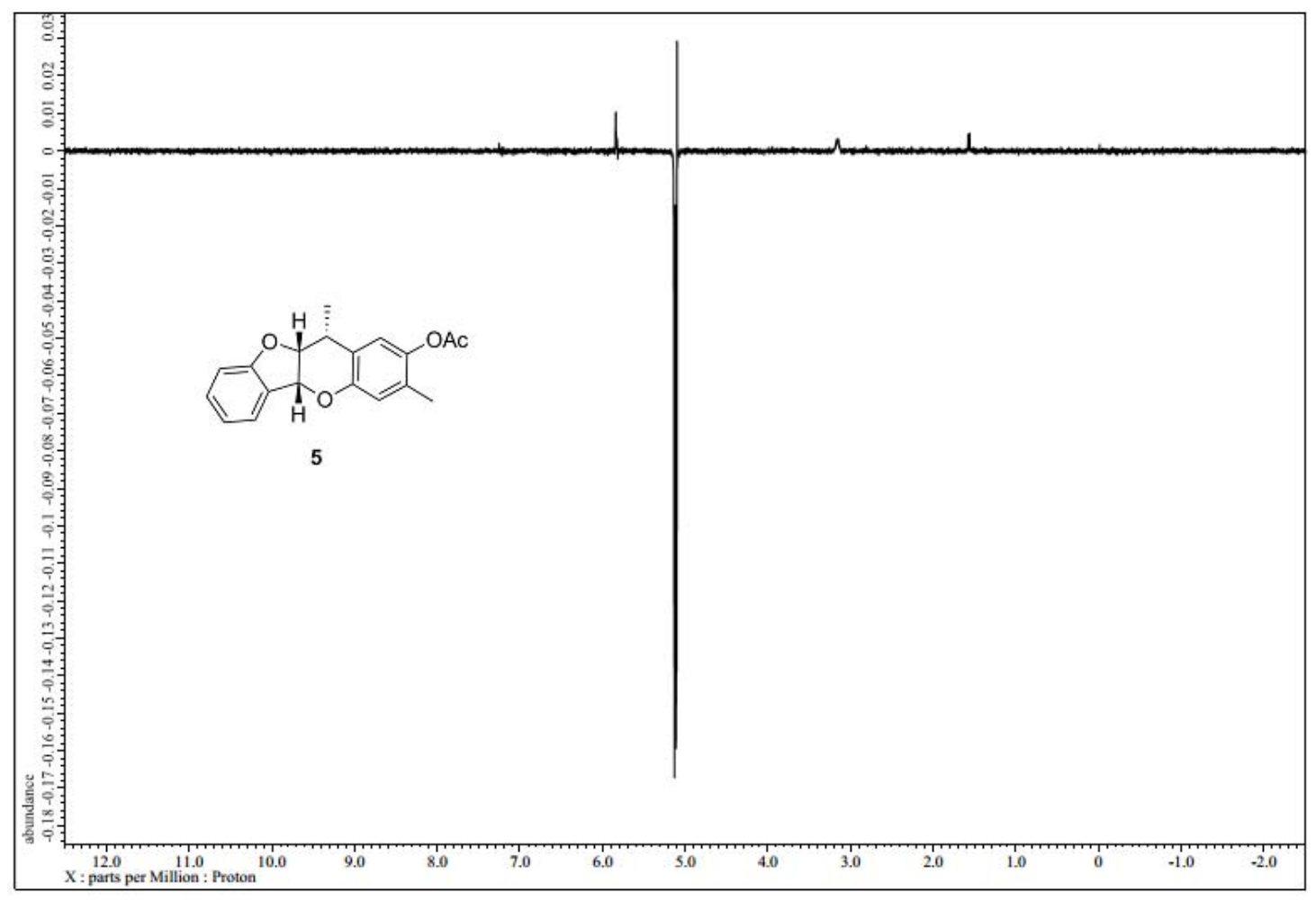

S26 


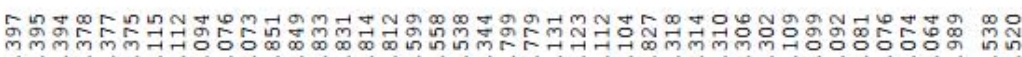

-

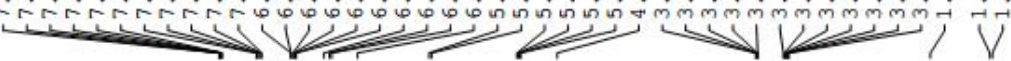

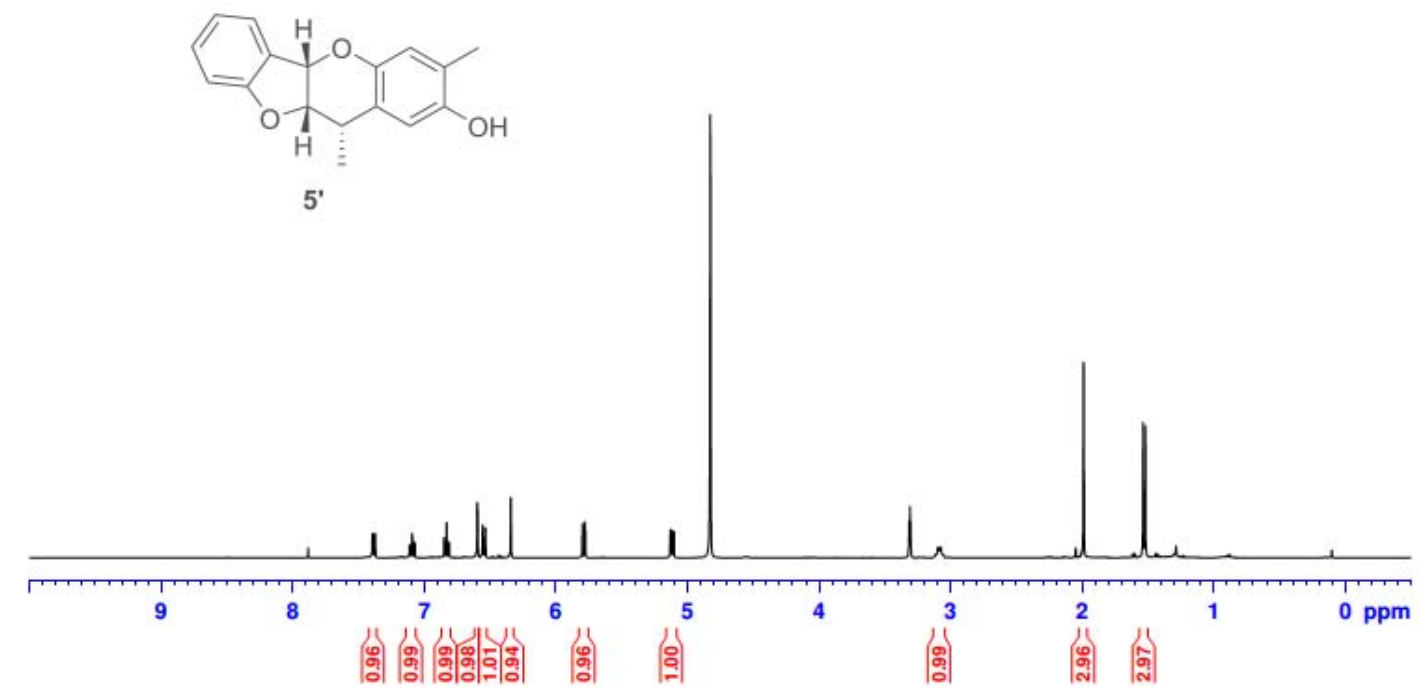

|
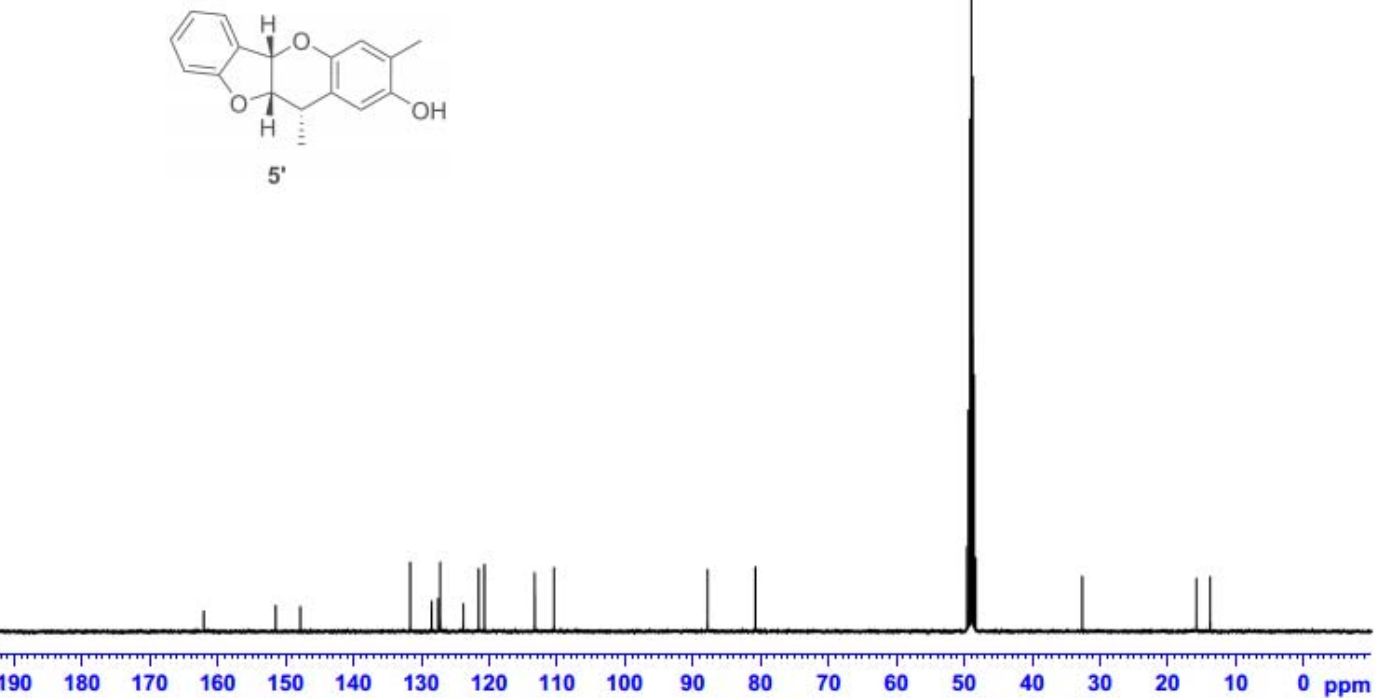

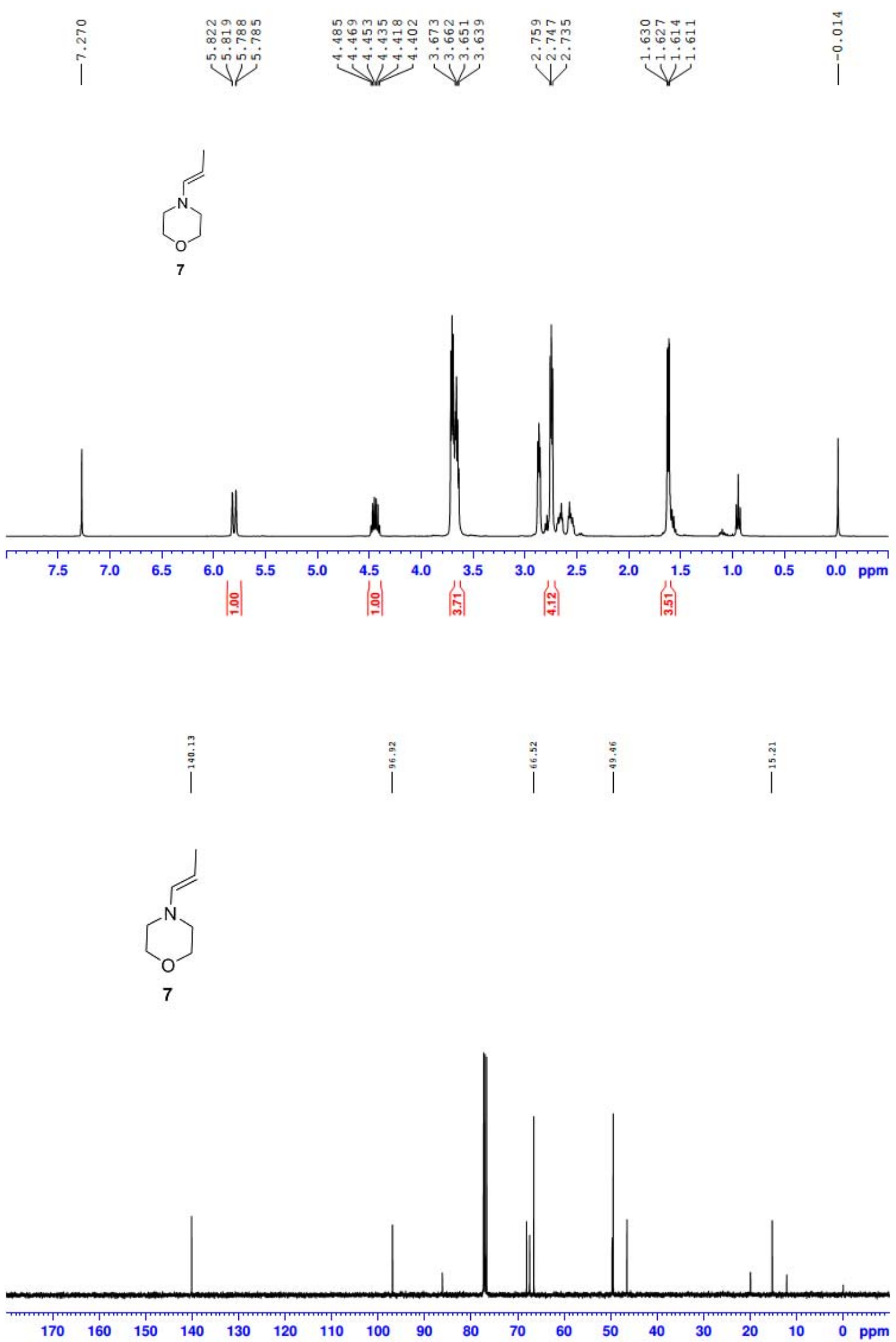


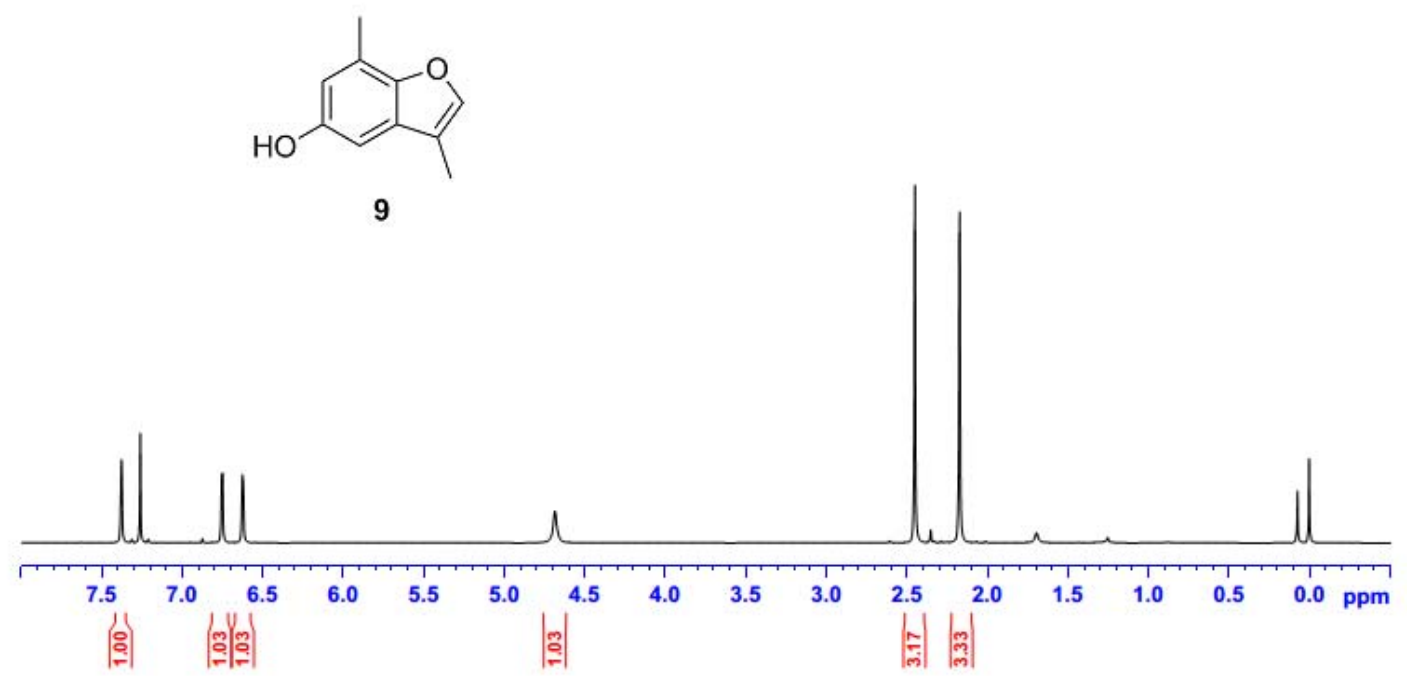<smiles>Cc1coc2c(C)cc(O)cc12</smiles>

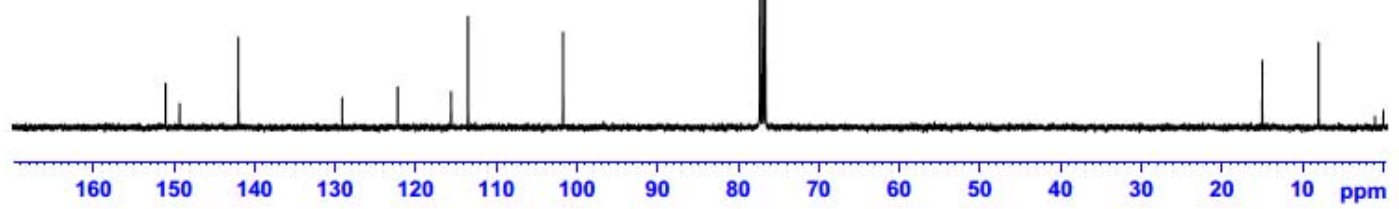




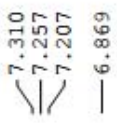

Vு

|

ii<smiles>Cc1cc2occ(C)c2cc1O</smiles>

10

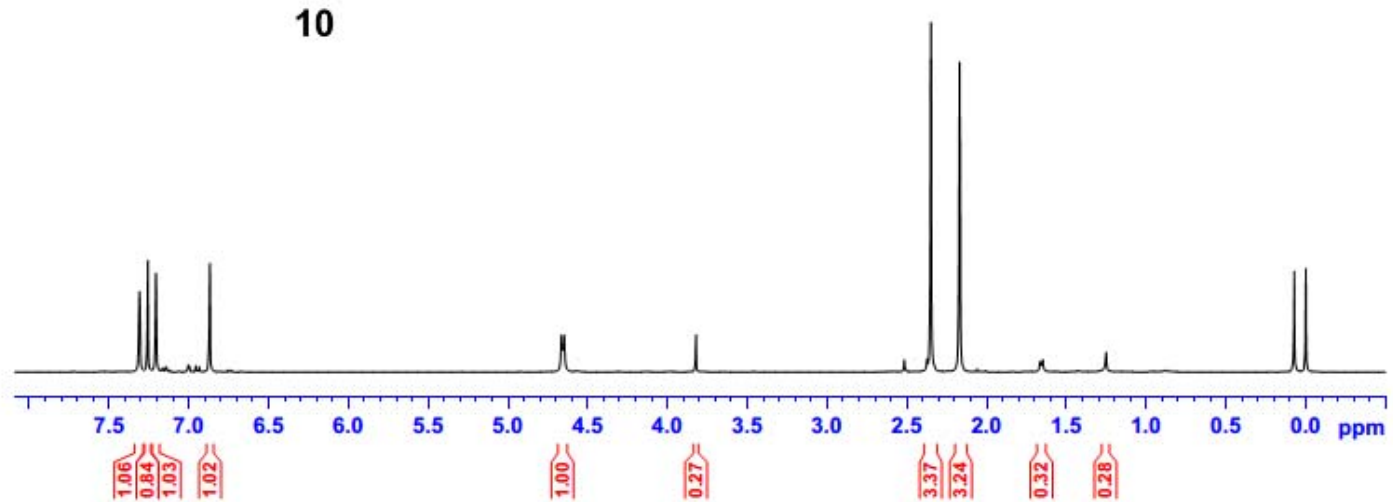

V|<smiles>Cc1cc2occ(C)c2cc1O</smiles>

10

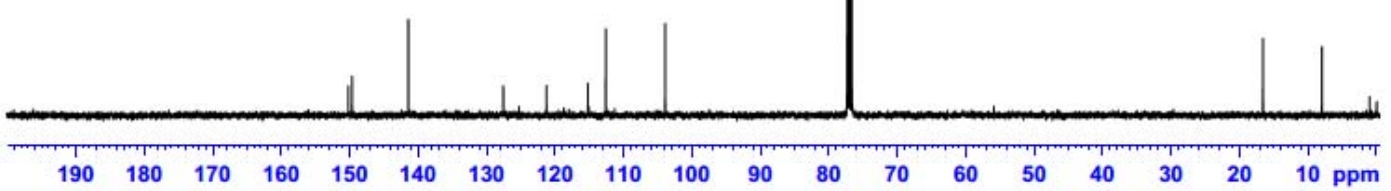




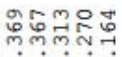

inisis

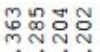

نิ่ง<smiles>CC(=O)Oc1cc2c(C)coc2cc1C</smiles>

11

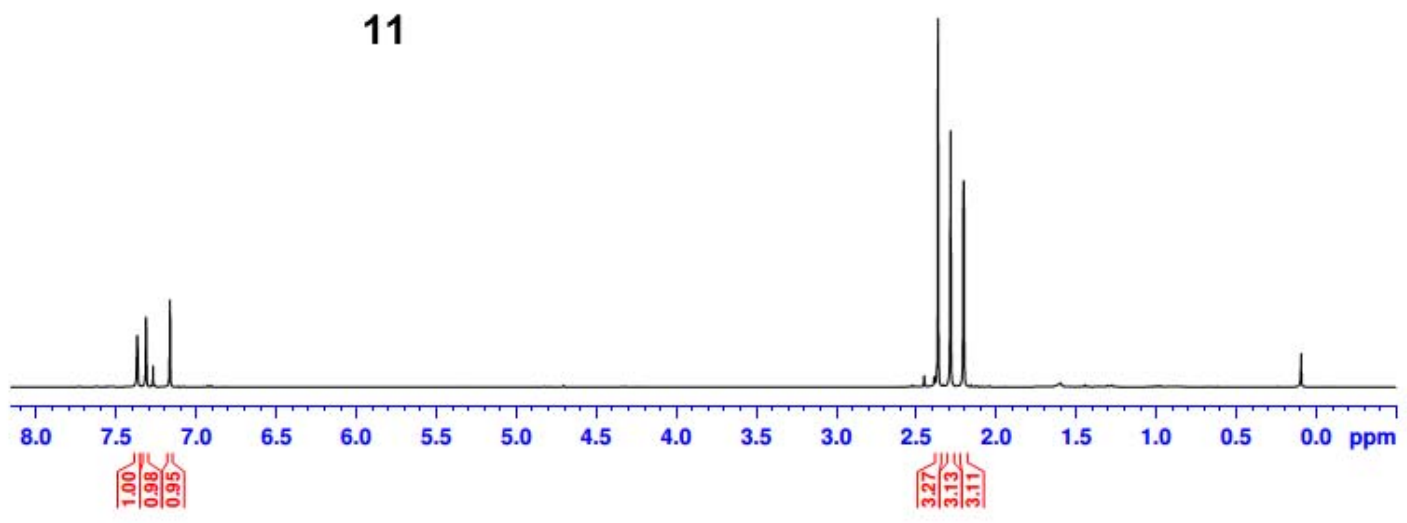<smiles>CC(=O)Oc1cc2c(C)coc2cc1C</smiles>

11

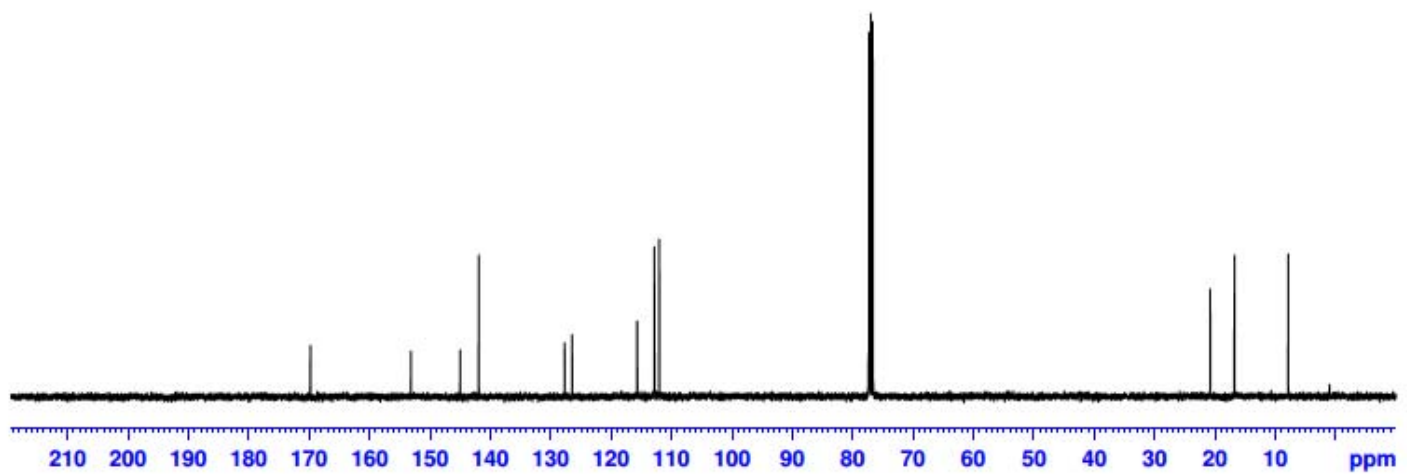


<smiles>Cc1cc2occ(CO)c2cc1O</smiles>

Paeoveitol D

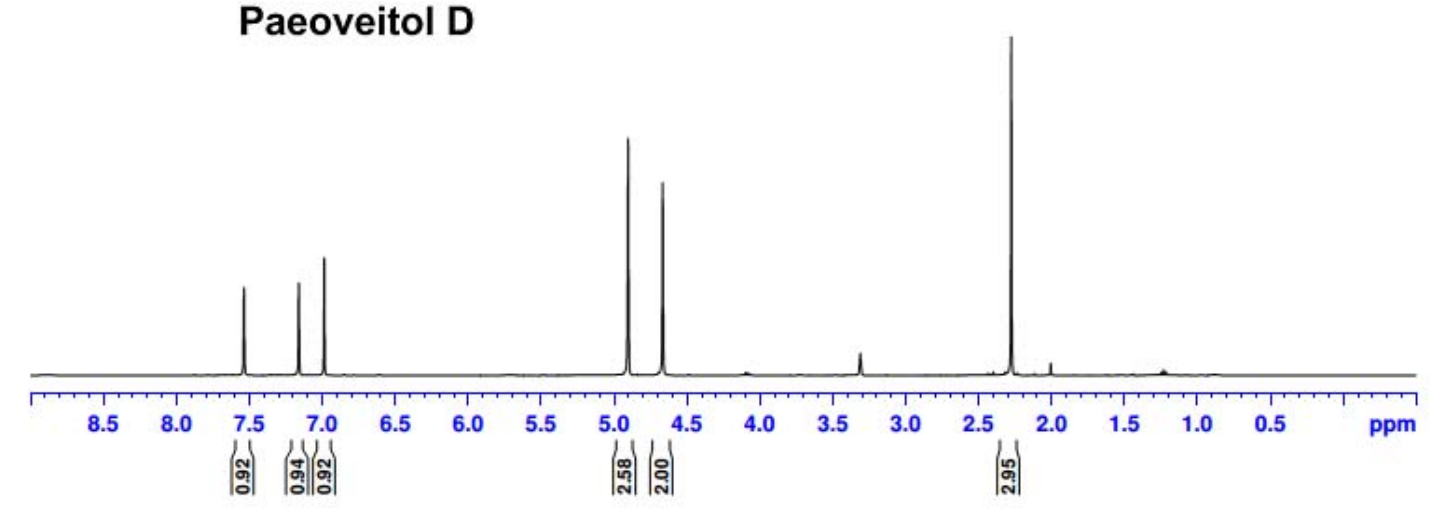

||

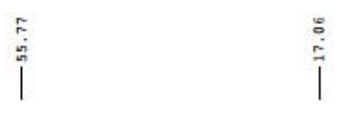<smiles>Cc1cc2occ(CO)c2cc1O</smiles>

Paeoveitol D

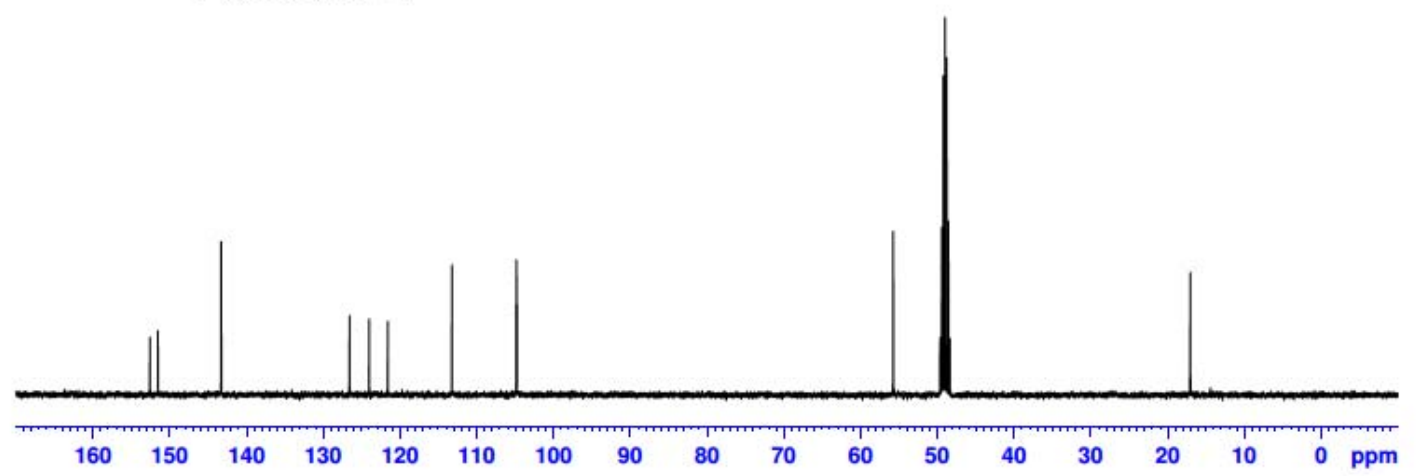




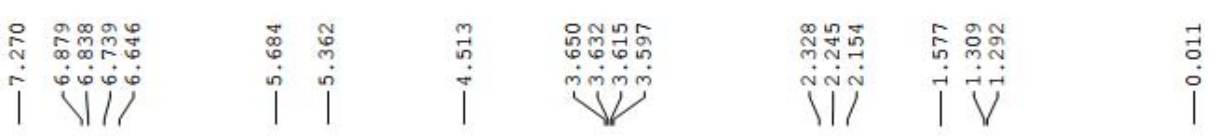
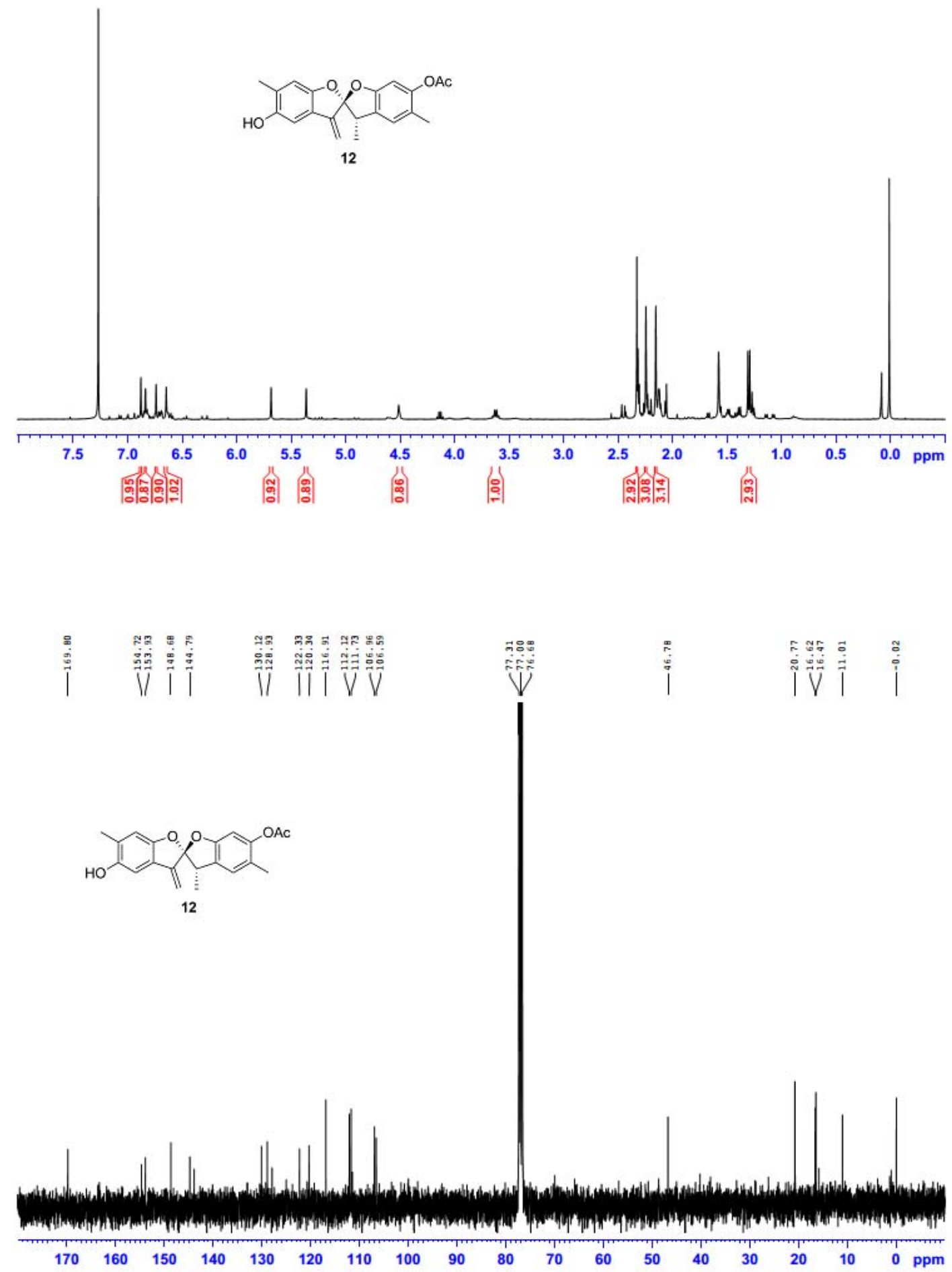


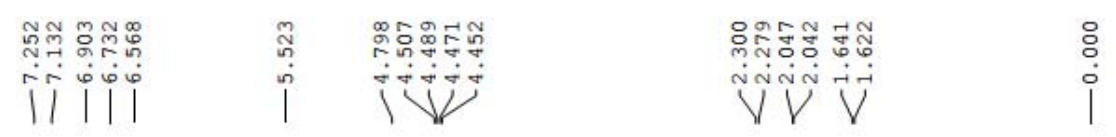
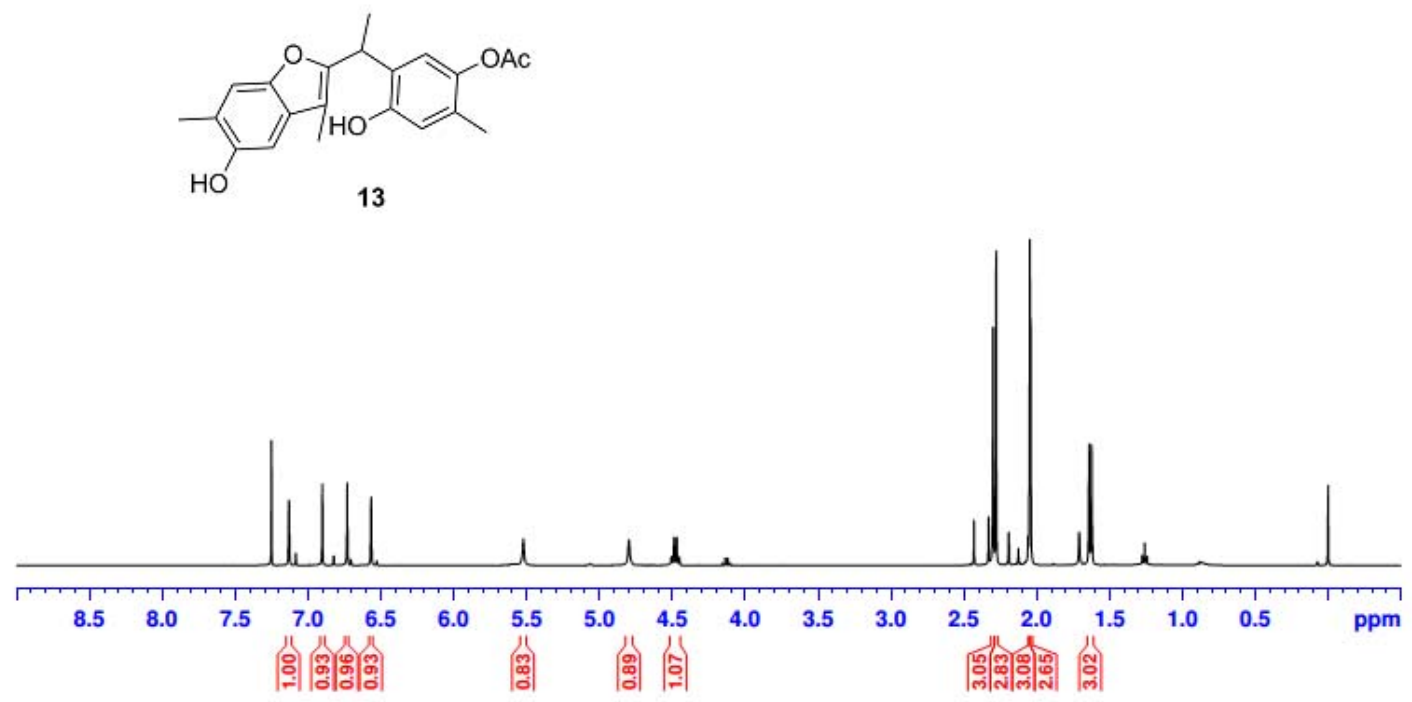

|

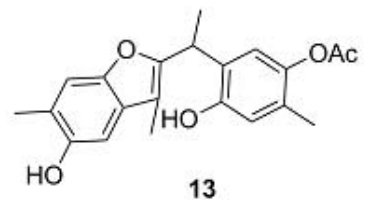

13

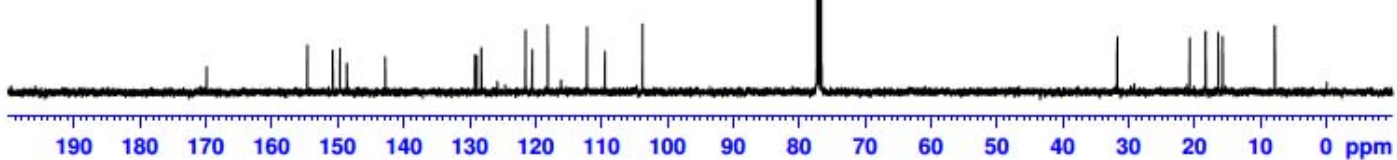



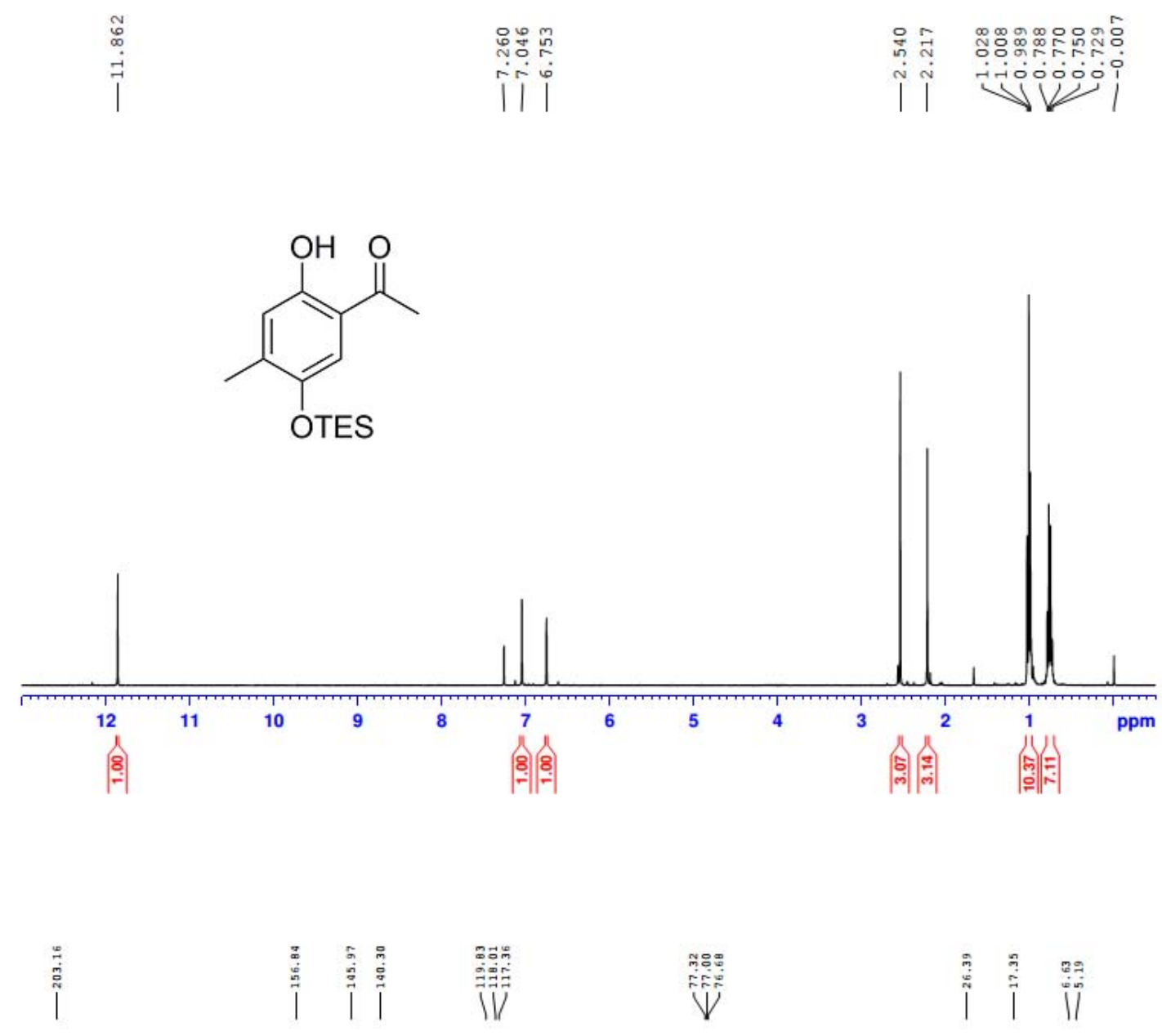<smiles>CC(=O)c1cc(OC=S)c(C)cc1O</smiles>

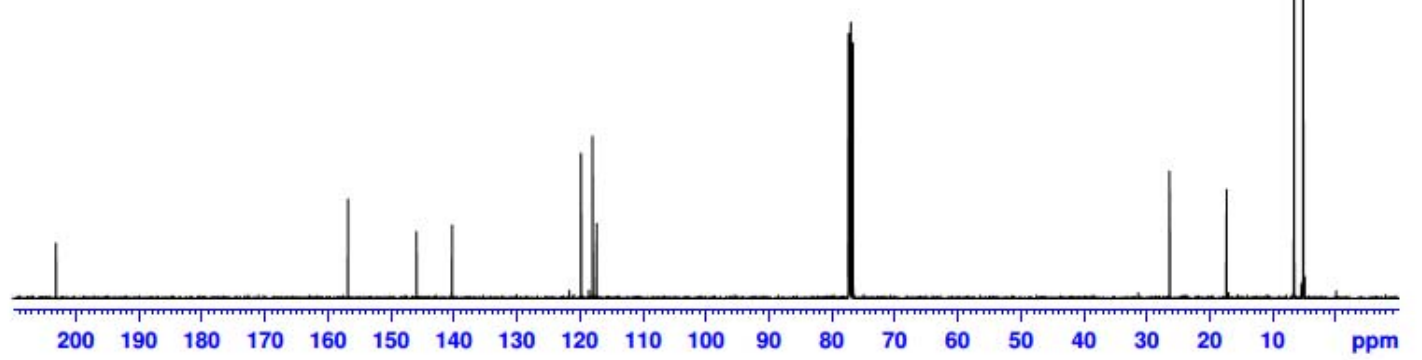


<smiles>COc1cc(C(C)O)c(O)cc1C</smiles>

14

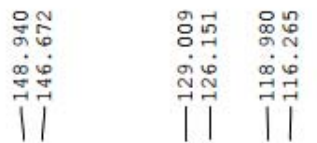

공ํำ

एवंश

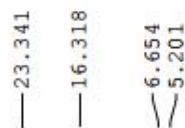<smiles>COc1cc(C(C)O)c(O)cc1C</smiles>

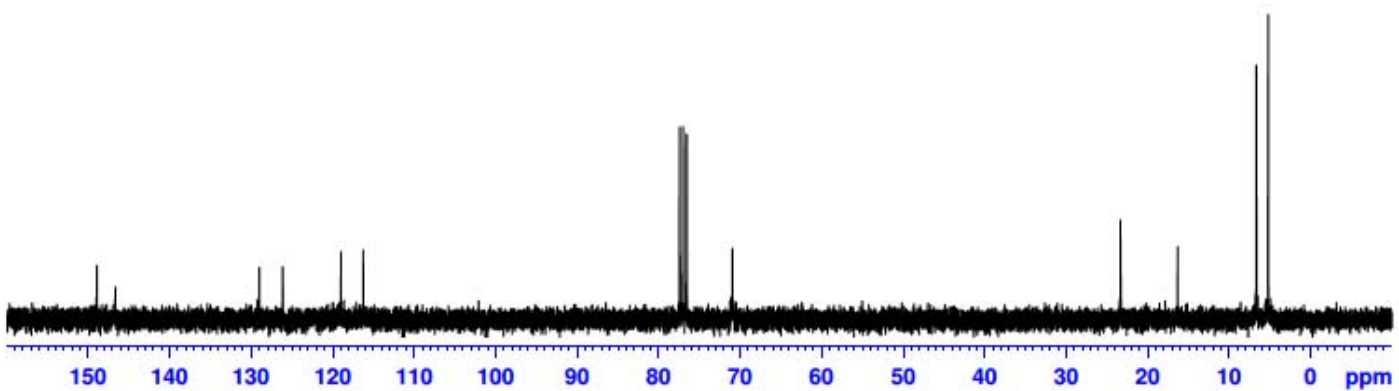



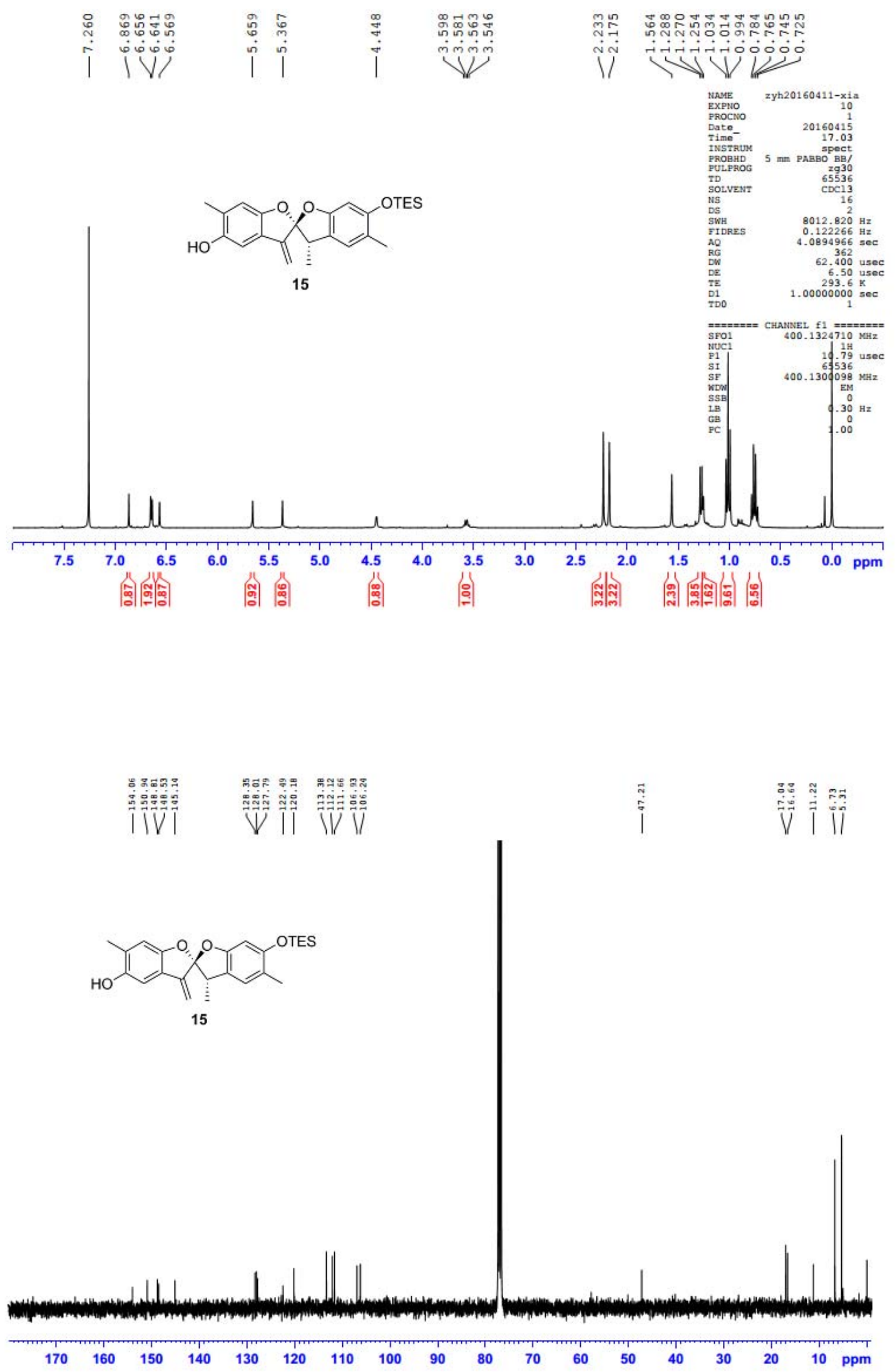

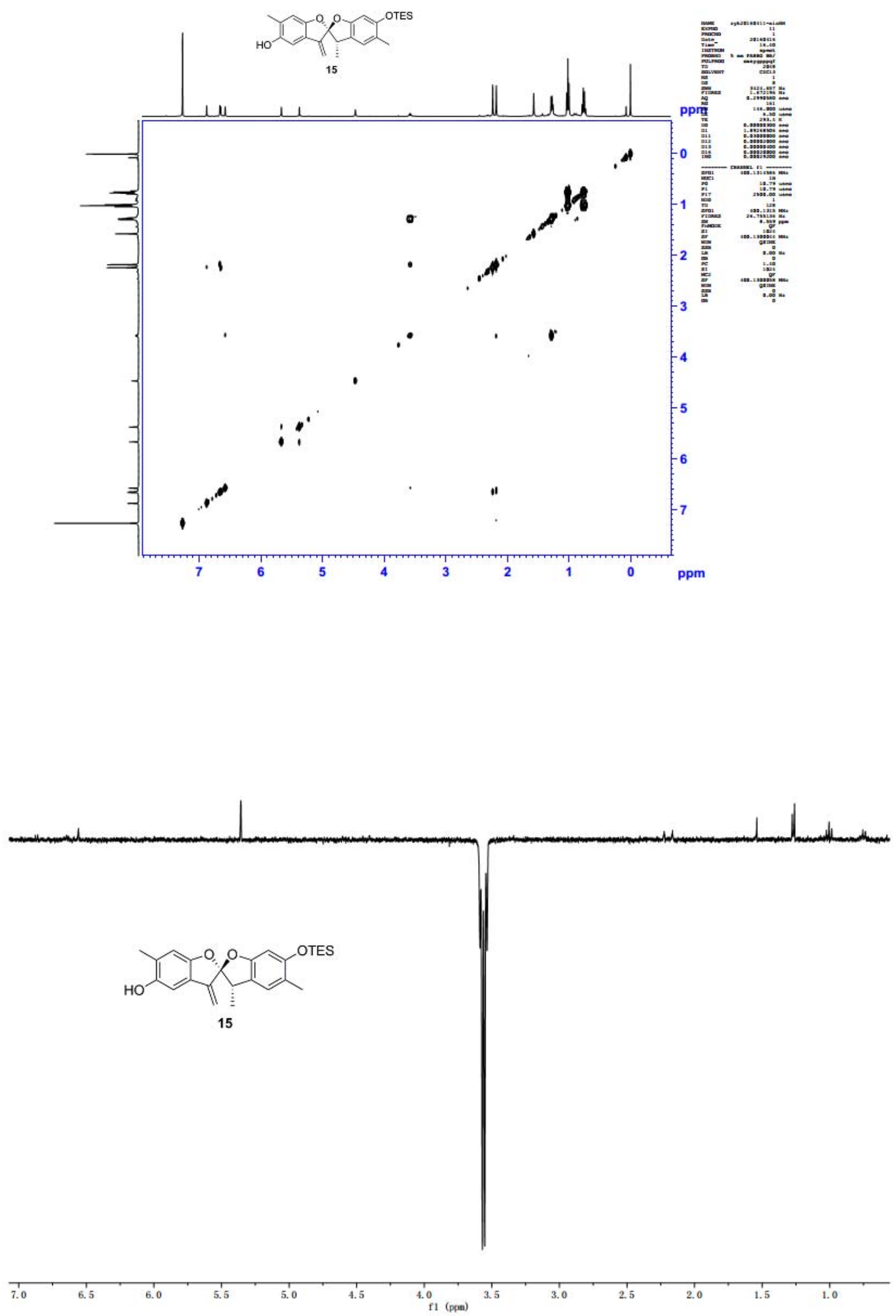

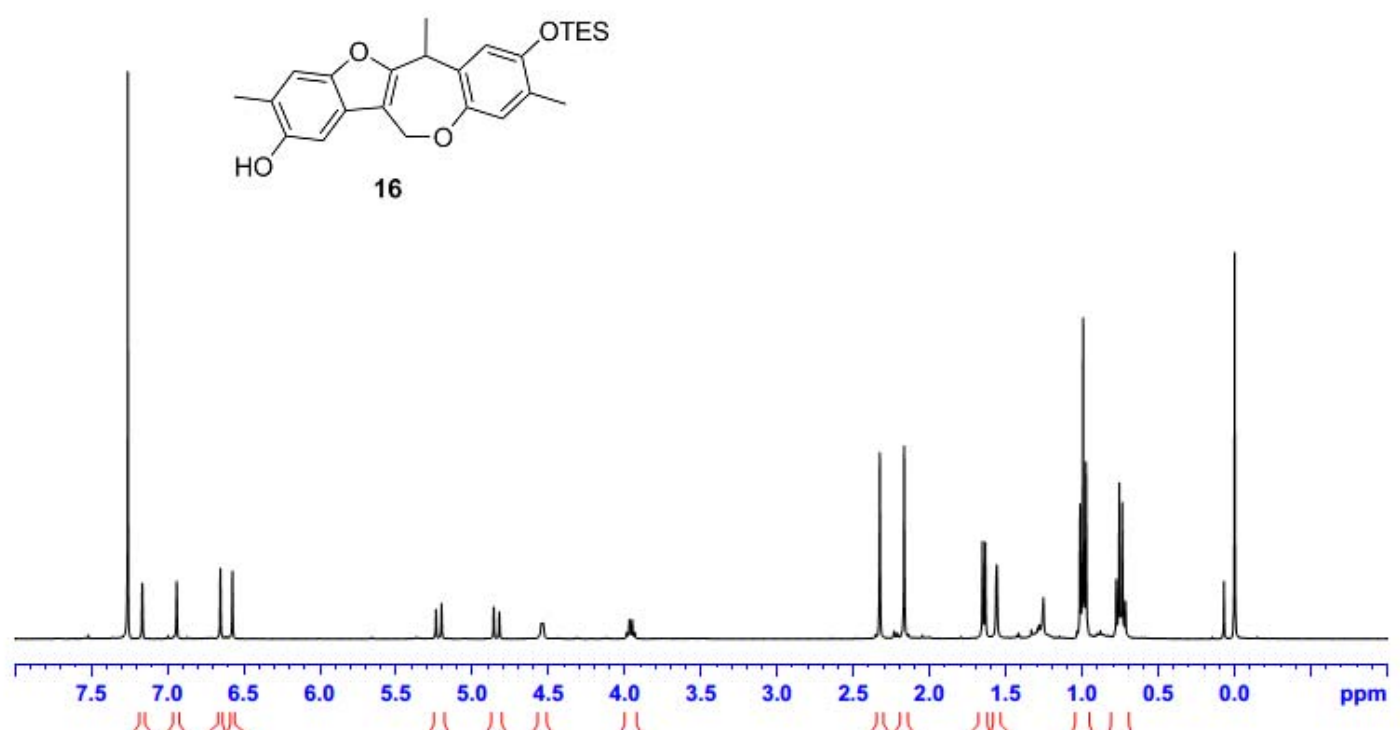

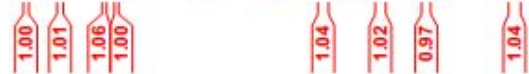

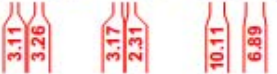
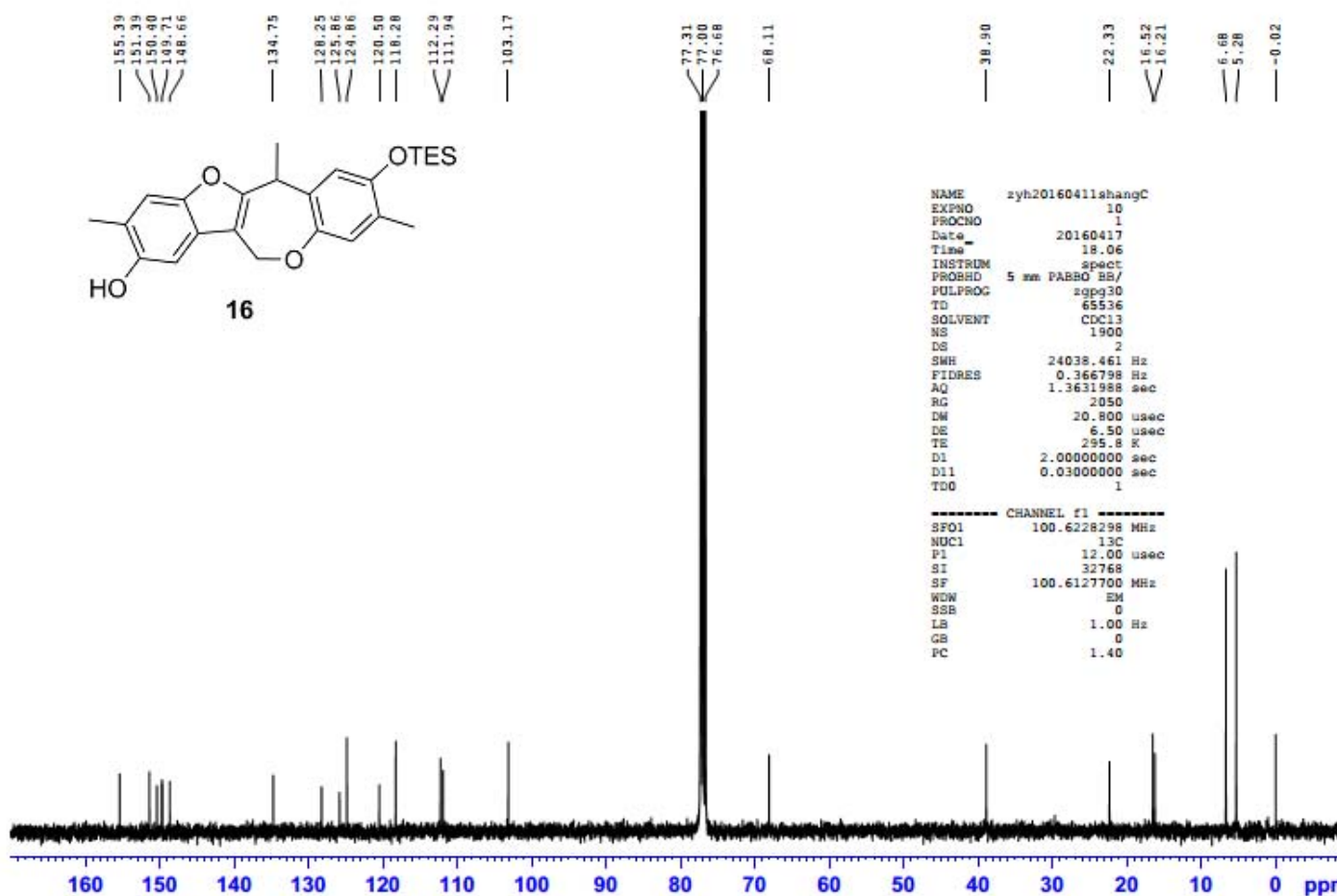

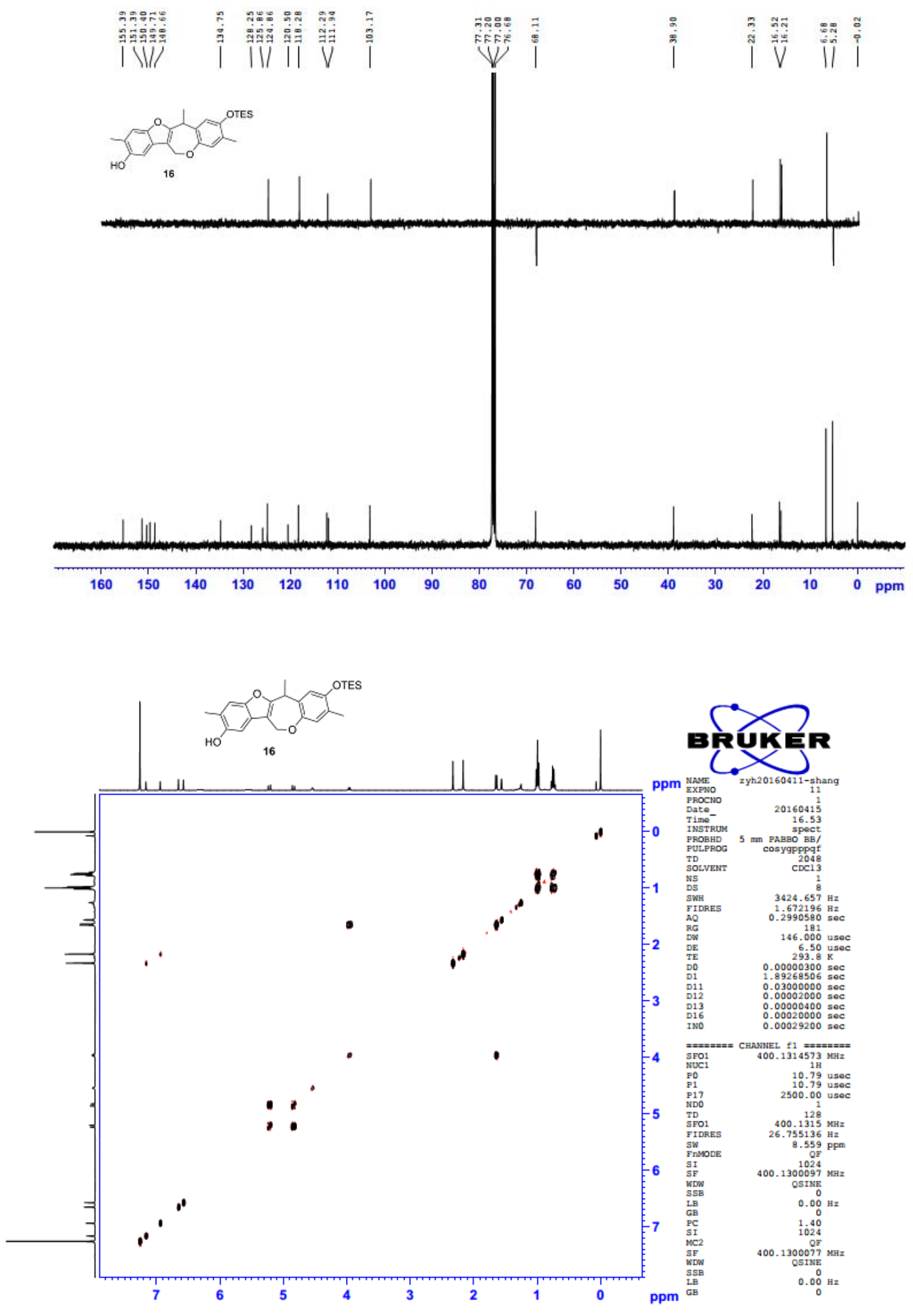


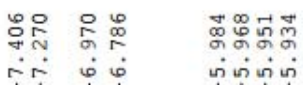

$\dot{i r i} \mid i$

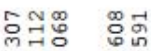

îंi

$\infty$
$\vdots$
0<smiles>CC(=O)Oc1cc(C(C)OC(C)=O)c(O)cc1I</smiles>

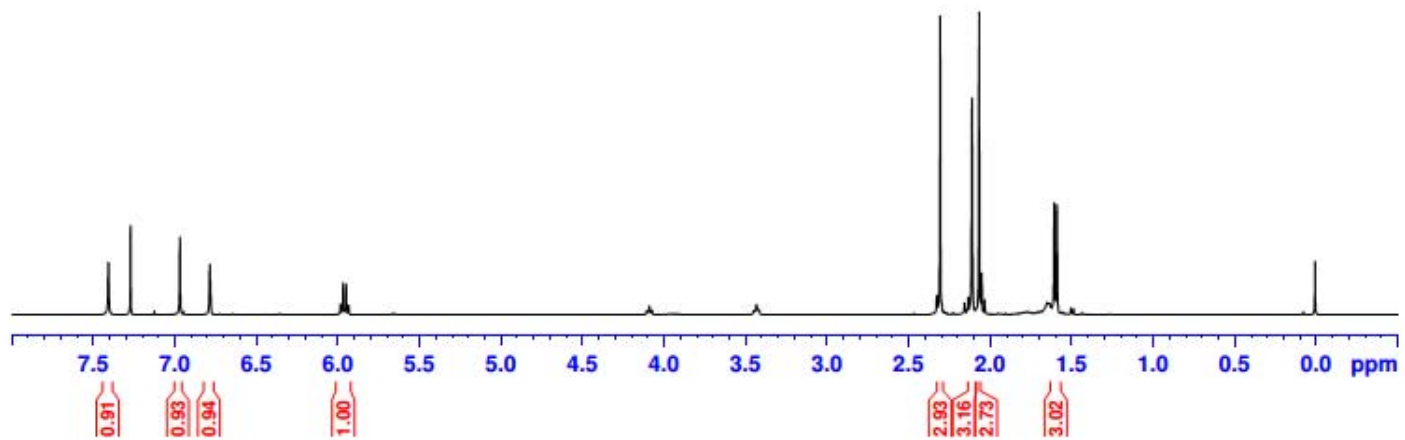

||

ฟ

Vิ|

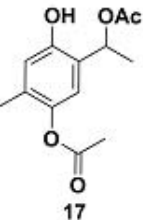




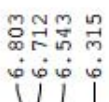

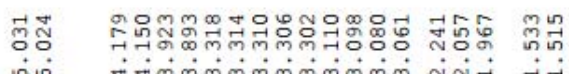

iviv

$\stackrel{0}{\stackrel{0}{0}}$
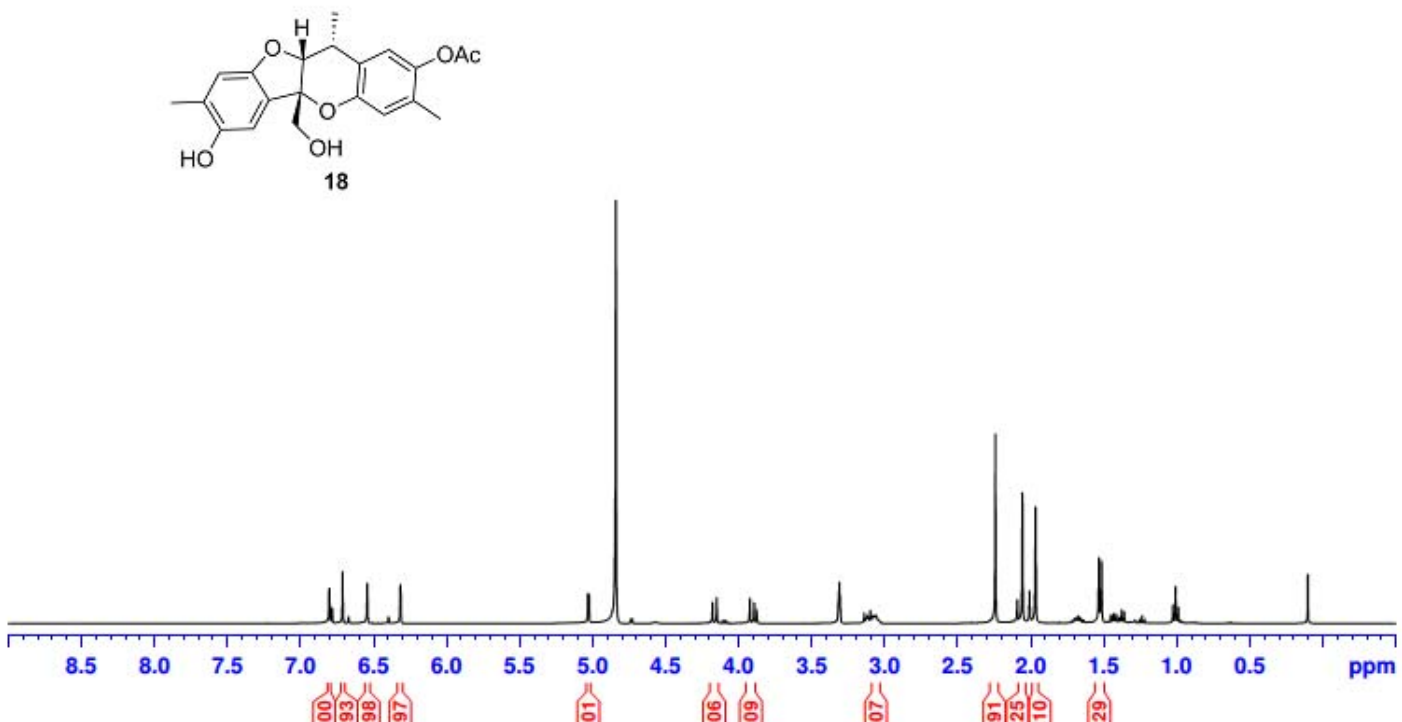

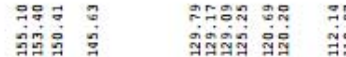

\|ी $\vec{V} \mid V \vec{V}$

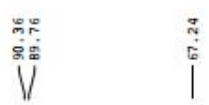

|
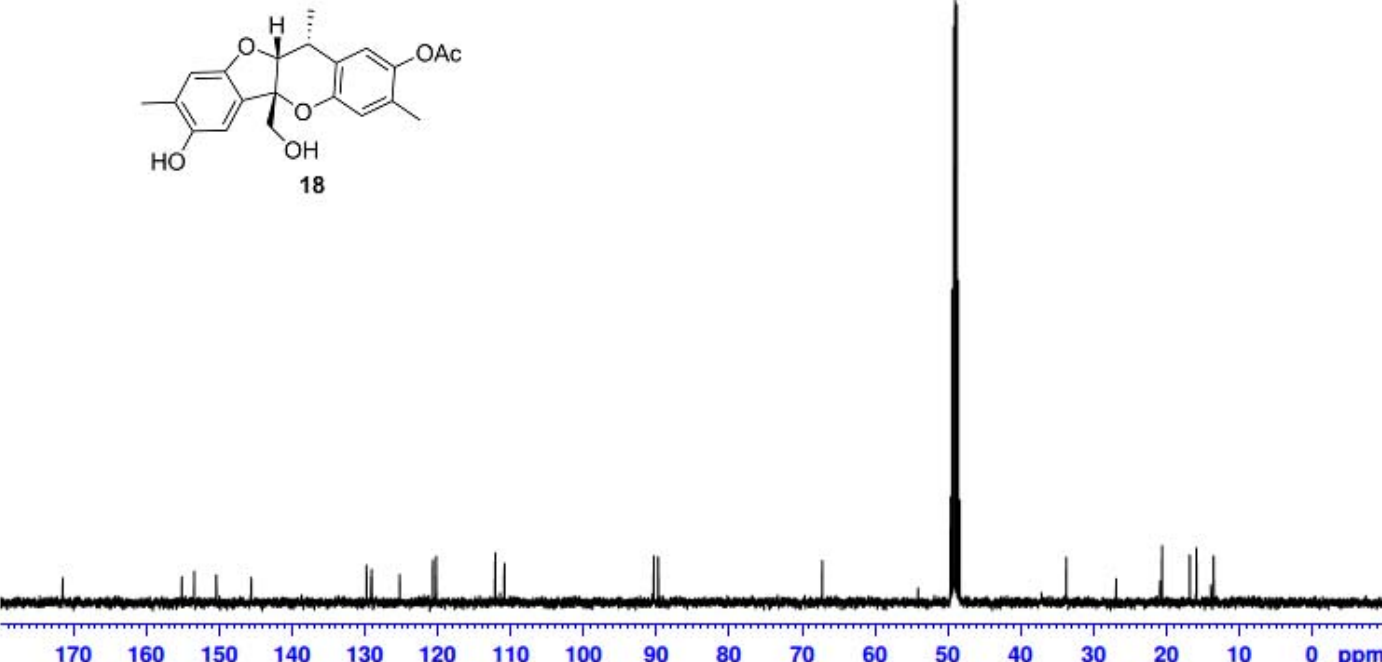


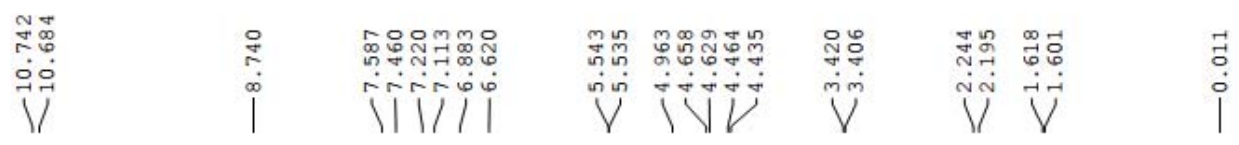
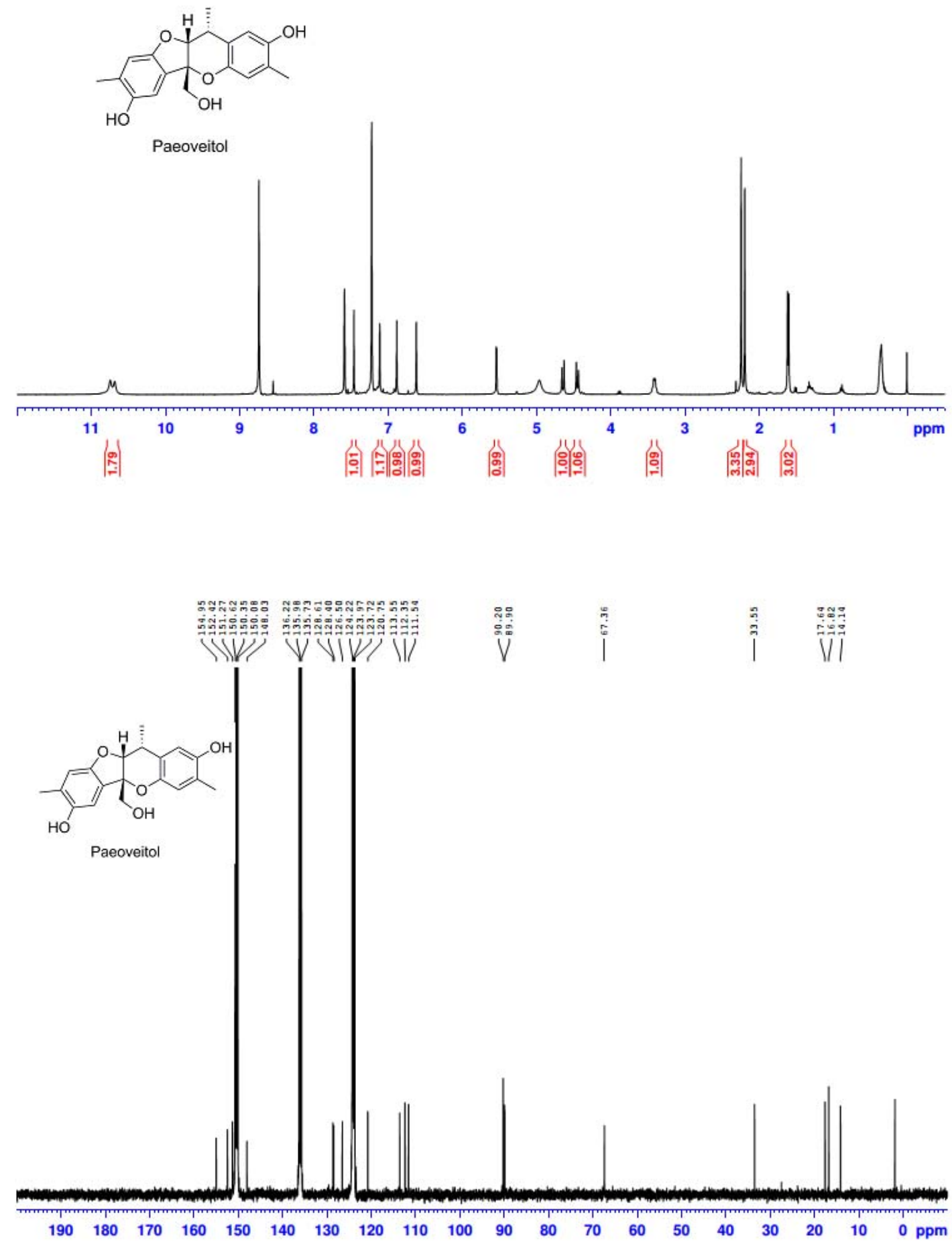
项目名称: Yu-hua Deng

用户名称：Breeze 用户 (Breeze)

\begin{tabular}{|c|c|c|c|}
\hline \multicolumn{4}{|c|}{ 样品信息 } \\
\hline 样品名称: & zyh-race2 IC3 & 采集者: & Breeze \\
\hline 样品类型: & 未知 & 样品组名称: & zyh \\
\hline 瓶号: & $1: A, 1$ & 采集方法组: & $09 \mathrm{ml}$ IPAvsHex 02vs07 \\
\hline 进样次数: & 1 & 处理方法: & zyh race IC3 \\
\hline 进样体积: & $5.00 \mathrm{ul}$ & 通道名称: & 214.0 纳米 \\
\hline 运行时间: & 30.0 Minutes & 处理通道注释: & PDA 214.0 纳米 \\
\hline $\begin{array}{l}\text { 采集时间: } \\
\text { 处理时间: }\end{array}$ & $\begin{array}{l}2016 / 8 / 13 \text { 16:36:15 CST } \\
2016 / 8 / 13 \text { 17:08:18 CST }\end{array}$ & & \\
\hline
\end{tabular}

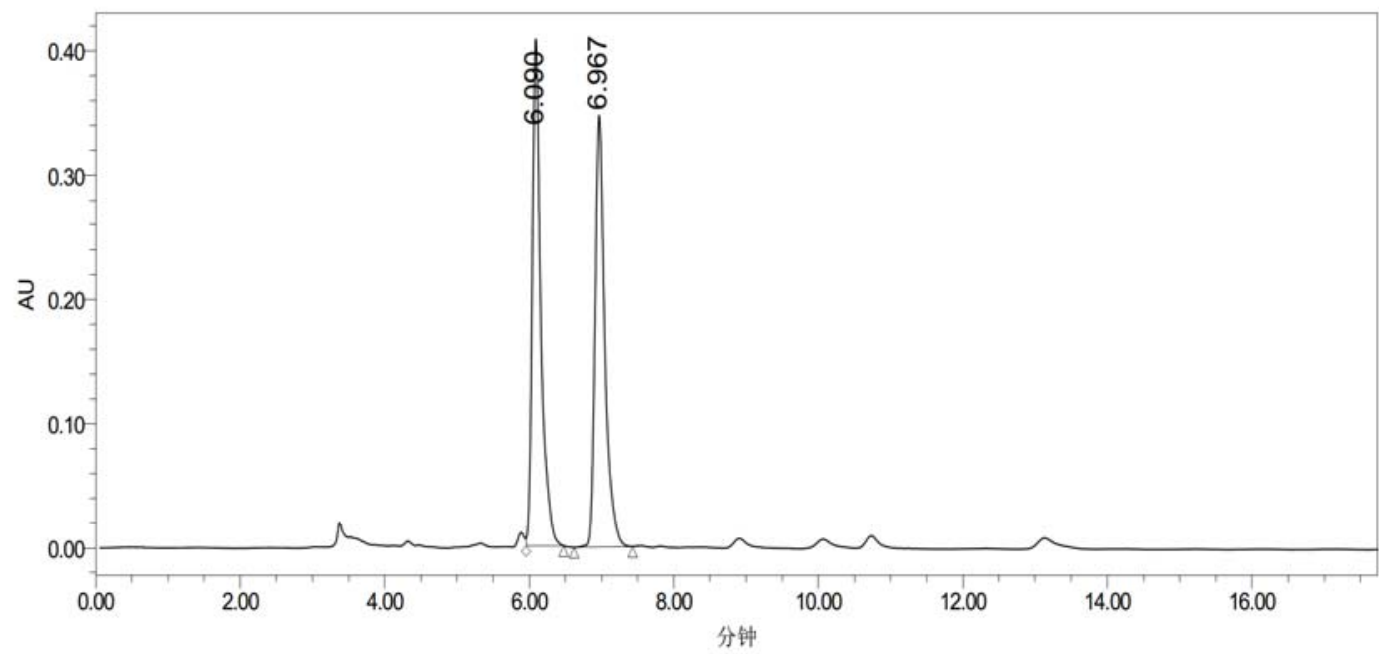

Channel: 2998; Channel Desc.: 2998 (190-400)纳米; Processing Method: zyh race IC3

名称:

\begin{tabular}{|c|c|c|c|c|c|}
\hline & 通道说明 & $\begin{array}{c}\text { 保留时间 } \\
\text { (分钟) }\end{array}$ & $\begin{array}{c}\text { 面积 } \\
\text { (微伏秒) }\end{array}$ & $\%$ 面积 & $\begin{array}{c}\text { 高度 } \\
\text { (微伏) }\end{array}$ \\
\hline 1 & 2998 (190-400)纳米 & 6.090 & 3360154 & 50.63 & 407858 \\
\hline 2 & 2998 (190-400)纳米 & 6.967 & 3275965 & 49.37 & 347660 \\
\hline
\end{tabular}


项目名称： Yu-hua Deng

\begin{tabular}{|c|c|c|c|}
\hline \multicolumn{4}{|c|}{ 样品信息 } \\
\hline 样品名称: & zyh-asy2 IC3 & 采集者: & Breeze \\
\hline 样品类型: & 未知 & 样品组名称: & zyh \\
\hline 瓶号: & $1: A, 3$ & 采集方法组: & $09 \mathrm{ml}$ IPAvsHex $02 \mathrm{vs} 07$ \\
\hline 进样次数: & 1 & 处理方法: & zyh asy IC3 \\
\hline 进样体积: & $5.00 \mathrm{ul}$ & 通道名称: & 214.0 纳米 \\
\hline 运行时间: & 60.0 Minutes & 处理通道注释: & PDA 214.0 纳米 \\
\hline $\begin{array}{l}\text { 采集时间: } \\
\text { 处理时间: }\end{array}$ & $\begin{array}{l}2016 / 8 / 13 \text { 16:03:16 CST } \\
2016 / 8 / 13 \text { 17:09:18 CST }\end{array}$ & & \\
\hline
\end{tabular}

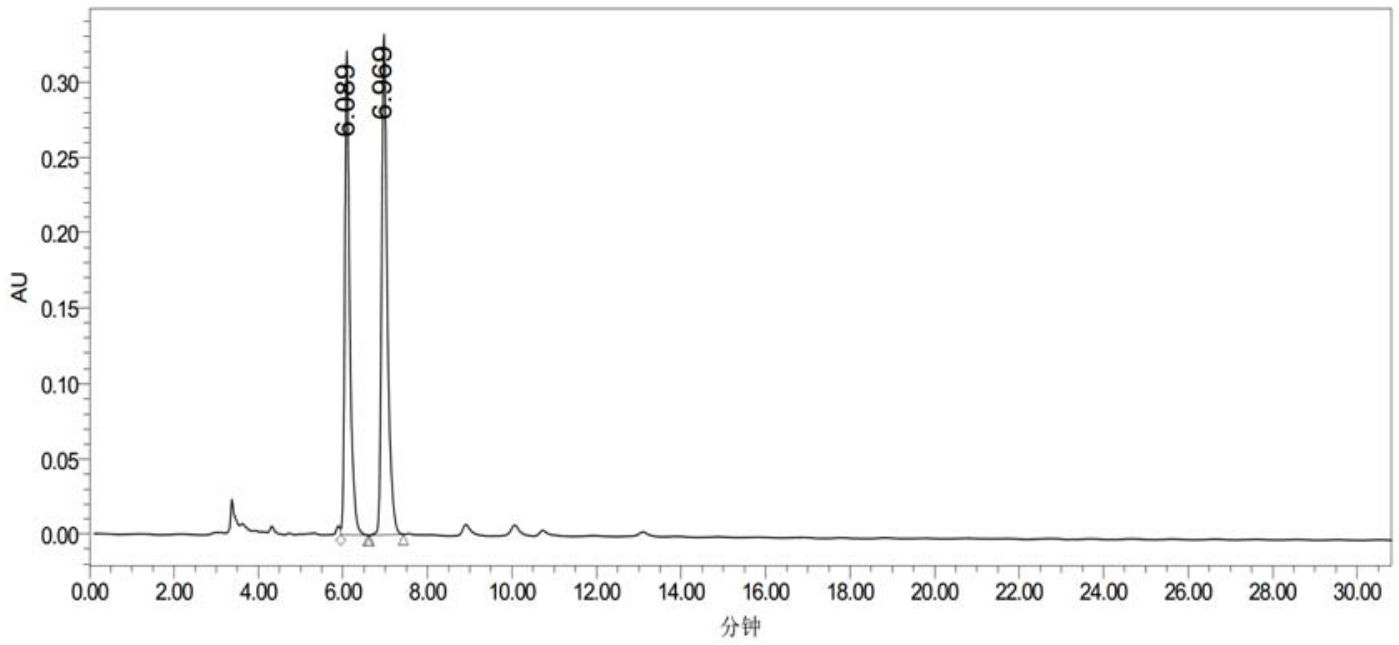

Channel: 2998; Channel Desc.: 2998 (190-400)纳米; Processing Method: zyh asy IC3

名称:

\begin{tabular}{|c|c|c|c|c|c|}
\hline & 通道说明 & $\begin{array}{c}\text { 保留时间 } \\
\text { (分钟) }\end{array}$ & $\begin{array}{c}\text { 面积 } \\
\text { (微伏*秒) }\end{array}$ & $\%$ 面积 & $\begin{array}{c}\text { 高度 } \\
\text { (微伏) }\end{array}$ \\
\hline 1 & 2998 (190-400)纳米 & 6.089 & 2620995 & 45.50 & 320830 \\
\hline 2 & 2998 (190-400)纳米 & 6.969 & 3139909 & 54.50 & 332190 \\
\hline
\end{tabular}

